\title{
nox2/cybb Deficiency Affects Zebrafish Retinotectal Connectivity
}

\author{
[CCory J. Weaver, ${ }^{1}{ }^{\circledR}$ Aslihan Terzi, ${ }^{1}$ Haley Roeder, ${ }^{1}$ Theodore Gurol, ${ }^{1}{ }^{\circledR}$ Qing Deng, ${ }^{1,2}$ Yuk Fai Leung, ${ }^{1,3}$ \\ and $\odot$ Daniel M. Suter ${ }^{1,3,4}$ \\ ${ }^{1}$ Department of Biological Sciences, ${ }^{2}$ Purdue Institute of Inflammation, Immunology and Infectious Disease, ${ }^{3}$ Purdue Institute for Integrative Neuroscience, \\ and ${ }^{4}$ Bindley Bioscience Center, Purdue University, West Lafayette, Indiana 47907
}

NADPH oxidase (Nox)-derived reactive oxygen species (ROS) have been linked to neuronal polarity, axonal outgrowth, cerebellar development, regeneration of sensory axons, and neuroplasticity. However, the specific roles that individual Nox isoforms play during nervous system development in vivo remain unclear. To address this problem, we investigated the role of Nox activity in the development of retinotectal connections in zebrafish embryos. Zebrafish broadly express four nox genes (nox1, nox2/cybb, nox 5, and duox) throughout the CNS during early development. Application of a pan-Nox inhibitor, celastrol, during the time of optic nerve (ON) outgrowth resulted in significant expansion of the ganglion cell layer (GCL), thinning of the $\mathrm{ON}$, and a decrease in retinal axons reaching the optic tectum (OT). With the exception of GCL expansion, these effects were partially ameliorated by the addition of $\mathrm{H}_{2} \mathrm{O}_{2}$, a key ROS involved in Nox signaling. To address isoform-specific Nox functions, we used CRISPR/Cas9 to generate mutations in each zebrafish nox gene. We found that $n o x 2 / c y b b$ chimeric mutants displayed ON thinning and decreased OT innervation. Furthermore, nox2/cybb homozygous mutants (nox2/cybb ${ }^{-1-}$ ) showed significant GCL expansion and mistargeted retinal axons in the OT. Neurite outgrowth from cultured zebrafish retinal ganglion cells was reduced by Nox inhibitors, suggesting a cell-autonomous role for Nox in these neurons. Collectively, our results show that Nox2/Cybb is important for retinotectal development in zebrafish.

Key words: axonal growth; NADPH oxidases; neuronal development; reactive oxygen species; retinal ganglion cell; zebrafish

\section{Significance Statement}

Most isoforms of NADPH oxidase (Nox) only produce reactive oxygen species (ROS) when activated by an upstream signal, making them ideal candidates for ROS signaling. Nox enzymes are present in neurons and their activity has been shown to be important for neuronal development and function largely by in vitro studies. However, whether Nox is involved in the development of axons and formation of neuronal connections in vivo has remained unclear. Using mutant zebrafish embryos, this study shows that a specific Nox isoform, Nox2/Cybb, is important for the establishment of axonal connections between retinal ganglion cells and the optic tectum.

\section{Introduction}

NADPH oxidase (Nox) are involved in reactive oxygen species (ROS)-mediated signaling in multiple biological processes

Received May 5, 2016; revised April 30, 2018; accepted May 13, 2018.

Author contributions: C.J.W. wrote the first draft of the paper; C.J.W., A.T., H.R., Q.D., Y.F.L., and D.M.S. edited the paper; C.J.W., A.T., H.R., Q.D., Y.F.L., and D.M.S. designed research; C.J.W., A.T., H.R., and T.G. performed research; T.G. and Q.D. contributed unpublished reagents/analytic tools; C.J.W., A.T., H.R., Y.F.L., and D.M.S. analyzed data; C.J.W., A.T., H.R., Y.F.L., and D.M.S. wrote the paper.

This work was supported by the National Science Foundation (Grant 1146944-IOS), the Purdue Research Foundation, and the Office of the Executive Vice President for Research and Partnerships, National Institutes of Health (NIH Targeted Initiative to D.M.S. and Grant R35GM119787 to Q.D.), and the Purdue Center for Cancer Research (NIH Grant P30CA023168 to Q.D.). Yuk Fai Leung was partially supported by a research grant from the International Retinal Research Foundation. We thank Brian A. Link for providing the $T g$ (ath5:GFP) transgenic line for this project, Tobias Dick (German Cancer Research Center) for the roGFP2-Orp1 construct, Wenbiao Chen (Vanderbilt University) for zebrafish CRISPR reagents, Wolfgang Driever (University of Freiburg) for the nestin construct, and James Lister (Virginia Commonwealth University) for the otx2 and pax6a constructs.
(Brown and Griendling, 2009; Yoo et al., 2011; Choi et al., 2012; Kajla et al., 2012; Spencer et al., 2013; Weaver and TaylorFishwick, 2013; Nayernia et al., 2014; Tauzin et al., 2014). Noxderived ROS have also been shown to mediate signaling in the nervous system (Nayernia et al., 2014; Wilson et al., 2017). Nox activity in the mouse brain is essential for neuronal plasticity in the hippocampus and long-term potentiation (Tejada-Simon et al., 2005; Kishida et al., 2006; De Pasquale et al., 2014). Studies using cultured rat hippocampal neurons have shown that Nox2 is critical for the establishment of neuronal polarity (Wilson et al., 
2015, 2016). Nox2 activity also regulates neurite outgrowth and actin dynamics in cultured neurons from Aplysia californica (Munnamalai and Suter, 2009; Munnamalai et al., 2014). In addition, Nox inhibition causes defects in rat cerebellar development (Coyoy et al., 2013) and reduced neurite outgrowth of cerebellar neurons (Olguin-Albuerne and Moran, 2015). Furthermore, neural stem cells retain a high level of ROS that is critical for their self-renewal and ROS production in these cells has been linked to Nox2 (Dickinson et al., 2011; Le Belle et al., 2011; Ren et al., 2015; Nayernia et al., 2017). Taken collectively, these studies show that Nox-derived ROS are critical components of normal signaling and development in the nervous system (Nayernia et al., 2014; Wilson et al., 2017). However, the detailed functions of individual Nox isoforms during normal nervous system development in vivo are not well understood.

The goal of the present study was to determine the role of specific Nox isoforms in the development of axonal tracts in the nervous system. We used zebrafish embryos because they are a well validated model system of vertebrate neurodevelopment and compatible with in vivo imaging, immunolabeling, and molecular genetics. Gene sequences for five Nox isoforms (nox1, nox 2 also called $c y b b$ in zebrafish, nox4, nox5, and $d u o x)$, as well for the cytosolic subunits regulating Nox1 and Nox2 ( $222 p h o x, p 47 p h o x$, p67phox, p40phox, NOXO1, NOXA1, and Rac) have been identified in the zebrafish genome (Kawahara and Lambeth, 2007; Kawahara et al., 2007; Rosowski et al., 2016). Because the nox4 gene is not well annotated in zebrafish, it was excluded from the current study. In zebrafish, Nox-derived ROS mediate leukocyte and macrophage recruitment and promote wound healing (Yoo et al., 2011, 2012; Tauzin et al., 2014). With respect to the zebrafish nervous system, one study found that DUOX is important for peripheral axon regeneration (Rieger and Sagasti, 2011). We have shown recently that zebrafish express nox 1 , nox $2 / c y b b$, nox 5 , and duox broadly throughout the CNS during early development, including in the retina (Weaver et al., 2016). However, whether specific Nox isoforms play a functional role in specific aspects of zebrafish nervous system development remains unclear.

Here, we report that Nox2 is critical for the development of retinotectal connections. We show that transient pharmacological Nox inhibition during early development leads to an expansion of the ganglion cell layer (GCL), thinning of the optic nerve $(\mathrm{ON})$, and decreased innervation of the optic tectum (OT) by RGC neurons. Whereas expression of nox1, nox2, p22phox, p47phox, p67phox, p40phox, NOXO1, NOXA1, and Rac has not been demonstrated in zebrafish retinal ganglion cell (RGC) neurons so far, these Nox isoforms and cytosolic subunits are expressed in developing mouse RGC neurons (Dvoriantchikova et al., 2012). To determine which Nox isoforms are involved in retinal development, we generated isoform-specific nox mutant fish using CRISPR/Cas9. We found that homozygous nox $2 / c y b b$ mutations caused an initial expansion of the GCL and mistargeted retinal axons in the OT. In addition, we showed that pharmacological Nox inhibition decreased neurite outgrowth in cultured zebrafish retinal neurons. Together, these results demonstrate a novel role for nox $2 / c y b b$ in the developing zebrafish visual system.

\section{Materials and Methods}

Zebrafish housing/breeding. All animal work and experimental protocols were approved by the Purdue Animal Care and Use Committee. Zebrafish (Danio rerio) of the $\mathrm{AB}$ or $\operatorname{Tg}($ ath $5: G F P)$ line were maintained according to standard procedures (Westerfield, 2000; Hensley and Leung, 2010). Parental fish were set in pairs and embryos were collected
15 min after breeding to obtain cohorts of the same stage. Embryos were maintained in $\mathrm{E} 3$ medium containing the following (in $\mathrm{mM}$ ): $5 \mathrm{NaCl}, 0.17$ $\mathrm{KCl}, 0.33 \mathrm{CaCl}_{2}$, and $0.33 \mathrm{MgSO}_{4}$ plus $10^{-5} \%$ methylene blue) at $28^{\circ} \mathrm{C}$ and staged before harvest (Kimmel et al., 1995).

Nox inhibitor treatment of live embryos. Embryos were dechorionated with sharp forceps at $\sim 24 \mathrm{~h}$ postfertilization (hpf). Embryos were then maintained at $28^{\circ} \mathrm{C}$ until they reached $32 \mathrm{hpf}$. E3 medium was then replaced with medium containing $2.5 \mu \mathrm{M}$ celastrol (Cayman Chemical) dissolved in DMSO $(0.1 \% \mathrm{v} / \mathrm{v}$ final concentration). Control embryos received E3 medium containing 0.1\% DMSO alone. Embryos were then returned to the incubator for $4 \mathrm{~h}$, after which they were washed extensively with fresh E3 medium. Embryos were then returned to the incubator and maintained in E3 medium containing $0.003 \%$ $N$-phenylthiourea (PTU).

Immunolabeling. Between 12 and $23 \mathrm{hpf}, 0.003 \%$ PTU (SigmaAldrich) in E3 medium was applied to embryos used for immunolabeling to inhibit melanization (Li et al., 2012). At the indicated stages, samples were anesthetized in $0.016 \%$ tricaine methanesulfonate (Sigma-Aldrich) and fixed overnight in $4 \%$ paraformaldehyde (PFA) $/ 1 \times$ PBS at $4^{\circ} \mathrm{C}$. Embryos were then washed with PBT $(1 \times$ PBS, $0.5 \%$ Triton X-100) at room temperature (RT). Next, samples were incubated in prechilled acetone for $30-50 \mathrm{~min}$, depending on age, at $-20^{\circ} \mathrm{C}$. They were then washed extensively in PBT at RT before being incubated with $10 \mu \mathrm{g} / \mathrm{ml}$ proteinase K at RT (Sigma-Aldrich). Proteinase K digestion times ranged from 10-30 min depending on the age of the samples. Embryos were then fixed in $4 \%$ PFA/1 $\times$ PBS for 30 min and washed extensively in PBT. Samples were then incubated with blocking solution (10\% goat serum in PBT; Sigma-Aldrich) for $2 \mathrm{~h}$ at RT. The monoclonal $\mathrm{zn}-8$ antibody raised against alcama (zn-8; Zebrafish International Resource Center; RRID: AB_10013774). To label all axons, a mouse anti-acetylated $\alpha$-tubulin antibody (Sigma-Aldrich; RRID:AB_477585) was used. Both primary antibodies were diluted 1:500 in blocking solution. Samples were then incubated in primary antibody solution overnight at $4^{\circ} \mathrm{C}$, washed extensively in PBT the following day, and the secondary antibody, goat antimouse IgG Alexa Fluor 488 (Thermo Fisher), was diluted 1:500 in blocking solution. The nuclear counterstain diamidino-2-phenylindole (DAPI; Thermo Fisher) was added to the secondary antibody solution at a 1:500 dilution. Samples were incubated overnight in secondary antibody solution at $4^{\circ} \mathrm{C}$. The following day, samples were washed extensively with $\mathrm{PBT}$ and stored at $4^{\circ} \mathrm{C}$ in the dark.

Immunolabeled samples were positioned on coverslips in $1 \%$ lowmelting-point agarose dissolved in $1 \times$ PBS. A microscope slide was prepared by filling a grease ring with $70 \%$ glycerol/1× PBS. A coverslip containing samples was then inverted onto the microscope slide and sealed onto the grease ring for imaging. $Z$-stacks were collected at $1 \mu \mathrm{m}$ intervals using a Zeiss LSM 710 inverted scanning confocal microscope equipped with a $40 \times$ LD C-Apochromat 1.1 numerical aperture (NA) W Corr M27 objective (Zeiss). Panels showing zn-8 and anti-acetylated $\alpha$-tubulin labeling were maximum intensity projections, whereas DAPI panels show a single optical section (Figure $1,4 \& 5$ ). Maximum intensity projections were created using ImageJ software (Schneider et al., 2012) and additional image processing was performed with Photoshop CS3 extended (Adobe).

Live imaging. $\operatorname{Tg}($ ath 5:GFP) embryos were used to visualize GCL and ON formation in live embryos (Poggi et al., 2005). Samples were anesthetized in $0.016 \%$ tricaine methanesulfonate (Sigma-Aldrich) and mounted in 1\% low-melting-point agarose on $35 \mathrm{~mm}$ glass-bottomed dishes. Dishes were then filled with E3 medium containing $1 \times$ tricaine. Images were acquired from the anterior perspective showing the eyes and optic chiasm on a Nikon TE2000 Eclipse microscope equipped with a $10 \times$ Plan Fluor 0.3 NA objective, an X-cite 120 metal halide lamp (EXFO), the appropriate fluorescence filter sets (Chroma), a Cascade II cooled EM-CCD camera (Photometrics), and MetaMorph 7.8 software (Molecular Devices). Dorsal images showing the optic tecta were collected on a Zeiss LSM 710 laser scanning confocal microscope using a $20 \times$ Plan-Apochromat 0.8 NA M2 objective (Zeiss). For multiday imaging, embryos were removed from agarose with a scalpel and forceps and maintained in 24 -well plates at $28^{\circ} \mathrm{C}$. The embryos were then remounted as described each subsequent day. 
Image analysis. Length and area measurements were performed on raw images using MetaMorph 7.8 software. ON thickness was measured 5-10 $\mu \mathrm{m}$ medial to the GCL. Two measurements were taken per embryo and averaged to produce single value per sample. GCL area was measured from single wide-field images in the case of $\operatorname{Tg}($ ath $5: G F P)$ imaging or max intensity projections from $101 \mu \mathrm{m}$ confocal optical sections centered on the $\mathrm{ON}$ in the case of $\mathrm{zn}-8$ labeling. Retinal areas were measured from phase images [ $\operatorname{Tg}($ ath5:GFP $)]$ or a single $1 \mu \mathrm{m}$ optical section from the DAPI channel centered in the ON (zn-8 labeling). GFP- or zn-8positive area measurements were divided by the corresponding total retinal area to obtain the values reported and, again, two values were averaged per embryo. Tectal area was measured from maximum intensity projections compiled from 25-301 $\mu \mathrm{m}$ optical sections. An example of the measured area of one tectum is shown in Figure 1I. Areas from the left and right tecta were averaged to produce a single value for each embryo. Line scans of zn- 8 fluorescent intensity in the optic tectum were performed with MetaMorph 7.8 software using raw, maximum intensity projections from $30-351 \mu \mathrm{m}$ optical sections through the midbrain. Lines were drawn from $\sim 10 \mu \mathrm{m}$ outside of the sample to a depth of $\sim 250$ $\mu \mathrm{m}$ (72 hpf) or $300 \mu \mathrm{m}$ (120 hpf) into the midbrain. Average intensity was collected from 25 pixels on either side of the line. Examples of the position of the line scans is shown in Figure 5, $A$ and $D$. Graphs of average intensity along the length of each line scan were produced using GraphPad Prism software version 6.05.

$\mathrm{H}_{2} \mathrm{O}_{2}$ rescue. $\mathrm{H}_{2} \mathrm{O}_{2}(2 \mathrm{~mm})$ dissolved in $\mathrm{E} 3$ medium was applied to zebrafish embryos immediately after celastrol washout as described above. Samples were maintained in $\mathrm{H}_{2} \mathrm{O}_{2}$-containing medium until harvest, with medium replaced every $12-16 \mathrm{~h}$. We chose not to coincubate celastrol and $\mathrm{H}_{2} \mathrm{O}_{2}$ due to possible complications arising from the oxidation of celastrol. Guide RNA (gRNA)-injected embryos were maintained in medium containing $2 \mathrm{mM} \mathrm{H}_{2} \mathrm{O}_{2}$ from injection to harvest for rescue experiments. The effects of prolonged exposure to $\mathrm{H}_{2} \mathrm{O}_{2}$ concentrations ranging from 1-5 mM were tested using $\operatorname{Tg}($ ath 5:GFP) embryos. We found that concentrations $<2.5 \mathrm{~mm} \mathrm{H}_{2} \mathrm{O}_{2}$ when applied alone did not noticeably affect embryonic survival, morphology, or ON outgrowth (data not shown). However, $\mathrm{H}_{2} \mathrm{O}_{2}$-treated embryos consistently showed more melanization compared with non- $\mathrm{H}_{2} \mathrm{O}_{2}$-exposed fish despite treatment with PTU. At later stages, this increased pigment caused dark spots in fluorescent images, as shown in Figure $3 \mathrm{H}$.

gRNA design and injection. gRNAs were designed with software developed at the Massachusetts Institute of Technology (http://crispr.mit. $\mathrm{edu} /$ ), which generates a list of scored candidate gRNAs as well as a list of scored potential off-target regions. Coding sequences from the each of the zebrafish nox genes were entered into the software to generate a list of candidate gRNAs. Each was then evaluated for their location in the target gene and the probability of off-target binding. Two gRNAs per isoform targeting $5^{\prime}$ regions of the coding sequences and with a score $\geq 95$ were identified (see Fig. $2 A$, Table 1). In addition, predicted offtarget sites for each of the selected gRNAs were analyzed. For a gRNA to be chosen, the predicted off-target sites had to meet the following criteria: score $\leq 0.5, \geq 2$ mismatches in the $11 \mathrm{nt}$ adjacent to the protospacer adjacent motif, $\geq 4$ mismatches overall, and $\leq 2$ predicted exonic offtargets. These strict parameters were chosen to minimize the probability of off-target mutagenesis. A summary of the predicted off-target sites for each gRNA is given in Table 2. We then used a set of zebrafish codon-optimized plasmid constructs to transcribe the gRNAs and Cas9 mRNA (Jao et al., 2013). The full protocol for plasmid construction is given at http://www.addgene.org/crispr/Chen. Forward and reverse oligos (Integrated DNA Technologies) matching the gRNAs shown in Table 1 were subcloned into the pT7-gRNA vector (available from Addgene; provided by Dr. Wenbiao Chen). gRNAs were synthesized using the MEGAshortscript T7 kit (Thermo Fisher). Cas9 was transcribed from the pCS2nls-Cas9-nls construct (provided by Dr. Wenbiao Chen) using the mMessage mMachine SP6 kit (Thermo Fisher). One nanoliter of a mixture containing 90-120 ng/ $\mu \mathrm{l}$ gRNA and $150 \mathrm{ng} / \mu \mathrm{l}$ Cas9 mRNA was injected into one-cell stage zebrafish embryos from a pair-bred $\mathrm{AB}$ cross. Injected embryos were incubated at $28^{\circ} \mathrm{C}$ until they reached the desired stage for genotyping.
Table 1. Summary of gRNAs targeting nox genes

\begin{tabular}{|c|c|c|c|c|c|}
\hline gRNA & Sequence $\left(5^{\prime}-3^{\prime}\right)$ & PAM & Score & Gene ID & Chromosome nol \\
\hline nox11 & GGGAAAGGGATTAAGGTAAG & TGG & 96 & & \\
\hline $\operatorname{nox} 1 \|$ & GGTTTAGTTTTCCACGGCGC & AGG & 98 & 555604 & 14 \\
\hline$n o \times 2 / c y b b 1$ & GGAAACTTTGCTGCAAATGA & AGG & 97 & & \\
\hline$n o \times 2 / c y b b \|$ & GGATTGGTGCTTCATGGAAT & CGG & 97 & 393386 & 11 \\
\hline nox51 & GGTGACCCATTCCAGCCAGC & GGG & 99 & & \\
\hline nox5 II & GGGAGATCGCACTCTCTCGC & AGG & 96 & 100149800 & 25 \\
\hline duox I & GGTGCTGATCCTCCGAGGGT & TGG & 95 & & \\
\hline duox II & GGCAGACTGGGACACGAGCA & CGG & 99 & 565097 & 25 \\
\hline
\end{tabular}

gRNA sequences are listed, along with the protospacer adjacent motif (PAM). Ideal PAM sites are NGG. Scores were assigned using design software (see Materials and Methods). Gene IDs refer to GenBank accession numbers.

Table 2. Summary of potential off-target sites for gRNAs

\begin{tabular}{llllll}
\hline gRNA & Sequence $\left(5^{\prime}\right.$-3') & PAM & Score & Gene ID & Chromosome no. \\
\hline nox1 I & AGGAAAGATATTAAGGTGAG & GAG & 0.2 & 567212 & 15 \\
nox1 II & GGTTTTGTTTCCCAGGCTC & CAG & 0 & 567798 & 2 \\
nox2/cybbI & GCAGACTTCCTCCAAATGA & AGG & 0.5 & 387260 & 8 \\
nox2/cybb II & GGATTGGGGCTTCGGGAGG & AGG & 0 & 393852 & 7 \\
nox5 I & GGTCTCCCACTCCTGCCAGC & AAG & 0.2 & 568565 & 20 \\
nox5 II & GTGAGATCGCAGTCCTCGA & AAG & 0.1 & 368662 & 2 \\
duox I & GATGCTGTCCTCCGATGGA & CAG & 0.3 & 563429 & 5 \\
duox II & GGGGGACTGCGAAACGAGCA & GAG & 0.5 & 368415 & 12 \\
\hline
\end{tabular}

Sequences for the predicted off-target sites are listed, along with the accompanying protospacer adjacent motif (PAM). Bold characters represent mismatches. Scores were assigned using design software (see Materials and Methods). Gene IDs refer to GenBank accession numbers and the chromosome on which each sequence appears is given.

Table 3. PCR primers used for genotyping

\begin{tabular}{|c|c|c|}
\hline gRNA & PCR primer sequence $\left(5^{\prime}-3^{\prime}\right)$ & Product length (bp) \\
\hline \multirow[t]{2}{*}{$\operatorname{nox} 11$} & ACA GTT TAG AGG GTG AAG AC & \multirow[t]{2}{*}{143} \\
\hline & CTC TGA CAG GAC TGG TTT A & \\
\hline \multirow{2}{*}{$\operatorname{nox} 1 \|$} & GTA CAC GCA TCA CCT CTT C & \multirow[t]{2}{*}{139} \\
\hline & TTG GAC GAA AGC GTC TG & \\
\hline \multirow[t]{2}{*}{$n o \times 2 / c y b b 1$} & CCC GAT AGC TTA CGA TAA CAA A & \multirow[t]{2}{*}{105} \\
\hline & CTC TCG ATC TCA TCT CCT GAT & \\
\hline \multirow[t]{2}{*}{ nox2/cybb II } & GAA TAG AAG CAA ACC CTC ACC & \multirow[t]{2}{*}{109} \\
\hline & GTC ATC CGA AGG TCC TAC TT & \\
\hline \multirow[t]{2}{*}{$\operatorname{nox} 51$} & AАTTTCACCCCTCACCATCC & \multirow[t]{2}{*}{903} \\
\hline & GACATGTTTCTTCTGACACAAC & \\
\hline \multirow[t]{2}{*}{$\operatorname{nox} 5 \|$} & AAT GTG ATG GAG CCA CTG & \multirow[t]{2}{*}{142} \\
\hline & CTA TAG ACC CAG ATG AGT TGC & \\
\hline \multirow[t]{2}{*}{ duox I } & TCT CTG GTC CGT CTG TATC & \multirow[t]{2}{*}{143} \\
\hline & ACG CTT CTG TTC TTG TGG & \\
\hline \multirow[t]{2}{*}{ duox II } & GTC TCC AAC TCT TCA CAGC & \multirow[t]{2}{*}{105} \\
\hline & GTA TAC ACG CAC CTG TGTC & \\
\hline
\end{tabular}

Genotyping. Genomic DNA was isolated from $72 \mathrm{hpf}$ tail biopsies (Wilkinson et al., 2013) or whole embryos (Meeker et al., 2007). Samples were dissolved in $50 \mathrm{~mm} \mathrm{NaOH}$ by incubating for $10 \mathrm{~min}$ at $95^{\circ} \mathrm{C}$. Onetenth volume of $1 \mathrm{~m}$ Tris $\mathrm{HCl}, \mathrm{pH} 8.0$, was added to neutralize and the samples were stored at $-20^{\circ} \mathrm{C}$. The amount of DNA was quantified using a NanoDrop Lite (Thermo Scientific). Genotyping was done using a gelbased method (Zhu et al., 2014). Briefly, gRNA target regions were amplified via PCR using the primers listed in Table 3. After PCR amplification with GoTaq Flexi (Promega), samples were heated to $95^{\circ} \mathrm{C}$ and allowed to cool for $10 \mathrm{~min}$ at RT. Samples were then loaded onto a $15 \%$ acrylamide gel without SDS and run for $2.5 \mathrm{~h}$ at $150 \mathrm{~V}$. Gels were stained with $50 \mu \mathrm{g} / \mathrm{ml}$ ethidium bromide (Sigma-Aldrich) for $10 \mathrm{~min}$ to visualize DNA bands. Heteroduplexes indicative of heterozygous mutants or chimeras exhibited lower electrophoretic mobility, as described previously (Zhu et al., 2014). In addition, we were able to reliably distinguish wild-type (WT) and homozygous mutants due to the electrophoretic shift as a result of DNA deletions. For Nox mutants, the gel-based genotyping method described here was verified with direct Sanger sequencing in the Purdue Genomics Core Facility to ensure reliability (see Fig. $4 A$ for mutant sequences). 
Generation of stable mutant lines. The injected and genotyped mutant zebrafish embryos were raised to adulthood. Adult injectants were outcrossed with an unrelated $\mathrm{AB}$ line and the resultant embryos were again genotyped via tail biopsy at $72 \mathrm{hpf}$ (Wilkinson et al., 2013). DNA from heterozygotes showing the formation of heteroduplexes on acrylamide gels (Zhu et al., 2014) was reamplified and purified with the Wizard SV Gel and PCR Clean-Up System (Promega). Purified DNA was then cloned into the pGEM-T Easy vector (Promega) for sequencing. Mutant sequences were confirmed with at least three technical replicates showing the same mutation from an individual larva. We then used the ExPASy translation tool (http://web.expasy.org/translate) to determine the resulting amino acid sequences and these were aligned with the WT protein sequences. Heterozygous larva carrying mutations leading to truncated Nox $2 / \mathrm{Cybb}$ proteins were used to found lines by outcrossing with $\mathrm{AB}$ strains to achieve a stable population of nox $2 / \mathrm{cybb}^{+/-}$adults. nox2/ $c y b b^{-1-}$ embryos were produced by crossing these heterozygous lines. Homozygous mutants, nox $2 / \mathrm{cybb}^{-1-}$, were identified via gel-based genotyping and/or Sanger sequencing.

In situ hybridization. The in situ hybridization probes fgf8, otx2, pax6a, and nestin were characterized previously (Mahler and Driever, 2007; Bishop et al., 2015). Probe containing vectors were linearized and riboprobes were synthesized using the DIG RNA labeling kit (Roche Diagnostics) and purified with $4 \mathrm{~m} \mathrm{LiCl}$ (Sigma-Aldrich). Whole embryos were anesthetized in $0.016 \%$ tricaine methanesulfonate (Sigma-Aldrich) at $36 \mathrm{hpf}$ before fixation in 4\% PFA in PBS. The samples were dehydrated stepwise in methanol $(30 \%, 50 \%, 70 \%$, and $100 \%)$, digested with 10 $\mu \mathrm{g} / \mathrm{ml}$ proteinase $\mathrm{K}$ (Thermo Fisher) for $12 \mathrm{~min}$, postfixed in $4 \%$ PFA for additional $30 \mathrm{~min}$, washed in $1 \times$ PBST $(1 \times$ PBS, $0.1 \%$ Tween 20$) 5$ times, and prehybridized in hybridization buffer $(50 \%$ formamide, $5 \times$ saline sodium citrate [SCC] $0.1 \%$ Tween $20,5 \mathrm{mg} / \mathrm{ml}$ torula yeast RNA, $50 \mu \mathrm{g} / \mathrm{ml}$ heparin) for $5 \mathrm{~h}$ at $65^{\circ} \mathrm{C}$. Embryos were then incubated in 100 ng of riboprobe in hybridization buffer overnight at $65^{\circ} \mathrm{C}$. Excess probes were washed with $50 \%$ formamide/ $2 \times$ SCCT (SSC, $0.1 \%$ Tween 20 ) twice, $2 \times$ SCCT once, and $0.2 \times$ SSCT twice. Embryos were washed 3 times in $1 \times$ PBST and incubated in blocking solution $(2 \mathrm{mg} / \mathrm{ml}$ bovine serum albumin, $2 \%$ normal sheep serum, $1 \times$ PBST) for $4 \mathrm{~h}$ at room temperature. Embryos were then incubated with an alkaline phosphatase-labeled sheep anti-digoxigenin antibody (Roche Diagnostics; RRID:AB_514497) diluted 1:3000 in blocking solution overnight at $4^{\circ} \mathrm{C}$ on a shaker. Excess antibody was removed by washing in PBST three times. Samples were then incubated in staining solution (100 mM Tris, pH 9.5, $50 \mathrm{~mm} \mathrm{MgCl}_{2}, 100 \mathrm{~mm} \mathrm{NaCl}, 0.1 \%$ Tween 20, $1 \mathrm{~mm}$ levamisol). For color detection, the samples were incubated in $0.3 \mathrm{mg} / \mathrm{ml}$ nitroblue tetrazolium and $0.15 \mathrm{mg} / \mathrm{ml}$ 5-bromo-4-chloro-3-indolyl-phosphate (Sigma-Aldrich) diluted in $100 \mathrm{~mm}$ Tris, $\mathrm{pH}$ 9.5, at room temperature on a shaker. Upon reaching the desired color intensity, the reaction was stopped by fixing samples in $4 \%$ PFA overnight at $4^{\circ} \mathrm{C}$. The next day, samples were washed 5 times in $1 \times$ PBST and then dehydrated stepwise in $30 \%, 50 \%, 70 \%$, and $100 \%$ methanol and incubated in benzyl alcohol/ benzyl benzoate solution (2:1) for 2 min to wash out nonspecific signals. Destained embryos were then rehydrated stepwise in $100 \%, 70 \%, 50 \%$, and $30 \%$ methanol and washed in $1 \times$ PBST 3 times. Labeled embryos were kept in $4 \%$ PFA at $4^{\circ} \mathrm{C}$. Whole-mount images of embryos were taken by mounting embryos in 3\% methylcellulose using the Olympus SZX16 stereomicroscope with an SDF PLAPO $1 \times$ PF objective. Images were captured by using the RT3 2.0Mp Slider CCD camera (SPOT Imaging Solutions). At the minimum, five individual samples were processed for each gene in each of the three experimental replicates.

Dissociated neuronal culture. The dissociated neuronal culture protocol was modified from Chen et al., 2013. At 33-34 hpf, embryos from the transgenic $\operatorname{Tg}($ ath 5:GFP) line were sterilized with 70\% ethanol and then transferred to a new dish with sterile E2 medium containing the following (in mM): $15 \mathrm{NaCl}, 0.5 \mathrm{KCl}, 1 \mathrm{MgSO}_{4}, 0.15 \mathrm{KH}_{2} \mathrm{PO}_{4}, 0.05 \mathrm{Na}_{2} \mathrm{HPO}_{4}$, $1.0 \mathrm{CaCl}_{2}$, and $0.7 \mathrm{NaHCO}_{3}$. Chorions were removed with sharp forceps and embryos were killed by decapitation. Whole eyes were extracted, triturated 20-30 times in L15 medium (Invitrogen) supplemented with $2 \%$ FBS, $0.4 \%$ penicillin/streptomycin, and $12.5 \%$ saline solution containing the following (in $\mathrm{mm}$ ): $10 \mathrm{D}$-glucose, $\mathrm{mm}$ Na-pyruvate, 1.26 $\mathrm{CaCl}_{2}$, and $32 \mathrm{HEPES}$ (Sigma-Aldrich). Individual cells were then plated on coverslips precoated with $0.5 \mathrm{mg} / \mathrm{ml}$ poly-D-lysine $(70-150 \mathrm{kDa})$ (Sigma-Aldrich) and $20 \mu \mathrm{g} / \mathrm{ml}$ laminin (Sigma-Aldrich). Cells were maintained in supplemented L15 medium overnight at RT.

Measurements of neurite outgrowth in cultured RGCs. For live-cell imaging, coverslips containing overnight retinal cultures were transferred from culture dishes to a custom-made open imaging chamber described previously (Suter, 2011). Before imaging, medium was replaced with serum-free L15. Imaging was performed with the Nikon TE2000 Eclipse microscope and Cascade II cooled CCD camera described above. A $60 \times$ 1.4 NA oil objective (Nikon) and an OG590 long-pass red filter (Chroma) were used for this set of experiments. RGCs were identified by positive GFP fluorescence, and initial differential interference contrast images of isolated neurons were acquired at time $=0 \mathrm{~min}$. After imaging, medium was replaced with L15 containing $0.1 \%$ DMSO (vehicle control) or different concentrations of the pan-Nox inhibitors celastrol or VAS2870 (Enzo Life Sciences). Additional images were acquired at 15 and $30 \mathrm{~min}$. Using MetaMorph 7.8 software, neurite lengths were measured from the base of the cell body to the tip of the growth cone lamellipodia on neurons with processes $\geq 15 \mu \mathrm{m}$. Neurite growth rates of individual cells were determined by subtracting the initial length from final length and dividing the growth difference by the elapsed time.

$\mathrm{H}_{2} \mathrm{O}_{2}$ imaging and quantification using roGFP2-orp1. roGFP2-orp1 mRNA was transcribed from pCS2 + vector using mMessage mMachine SP6 kit (Thermo Fisher). One nanoliter of $100 \mathrm{ng} / \mu \mathrm{lmRNA}$ was injected into one-cell-stage embryos. Injected embryos were incubated at $28^{\circ} \mathrm{C}$ until they reached desired stage. At $48 \mathrm{hpf}$, embryos were anesthetized in $0.016 \%$ tricaine and mounted in $1 \%$ low-melting-point agarose on 35 $\mathrm{mm}$ glass bottom dishes and dishes were filled with fish water containing tricaine. Images were acquired from anterior perspective on a Zeiss LSM 710 laser scanning confocal microscope using $20 \times$ Plan-Apochromat 0.8 NA M2 objective. roGFP2-orp1 was excited with 405 and 488 excitation and corresponding emission was acquired with a 500-520 band-pass filter. Time-lapse images were acquired over $20 \mathrm{~min}$ on a single optical section. $\mathrm{H}_{2} \mathrm{O}_{2}(20 \mathrm{~mm})$ was added after taking the first image and $2.5 \mathrm{~mm}$ dithiothreitol (DTT) was added after $10 \mathrm{~min}$ of imaging. To measure basal levels of $\mathrm{H}_{2} \mathrm{O}_{2}$ in WT and mutant embryos, $z$-stacks were acquired with $5 \mu \mathrm{m}$ section thickness. $z$-stacks were analyzed by ImageJ and the middle plane of each fish was selected to measure $\mathrm{H}_{2} \mathrm{O}_{2}$ levels. For analysis of $\mathrm{H}_{2} \mathrm{O}_{2}$ levels in RGC growth cones, roGFP2-orp1-expressing zebrafish embryos were selected for fluorescence at stage $\sim 28 \mathrm{hpf}$ and then killed via decapitation at $34 \mathrm{hpf}$. Retinas were dissected out and cells were plated as described above. Cultured RGC neurons were imaged using a TE2000 Eclipse microscope, a $60 \times 1.4$ NA oil objective (Nikon), and an iXon Ultra 888 EM CCD camera (Andor). roGFP2-orp1 was excited with $405 / 20$ and $480 / 30 \mathrm{~nm}$ excitation filters and corresponding emission was acquired at 535/30 nm using the dichroic mirror 505DCXR (R400/15 and 480/30, T510-700 nm; Chroma). Before imaging, the growth medium was replaced with serum-free, phenol red-free medium. An initial image was taken before treatment and then another set of images was taken 30 min after treatment with $100 \mu \mathrm{M} \mathrm{H}_{2} \mathrm{O}_{2}$.

To create ratiometric images of fish embryos, both 405 and 480 excitation images were opened in ImageJ. An average background value was subtracted from each channel and the intensity values of the 480 channel were added by " 1 " to eliminate pixel values of zero. Then, the 405 image was divided by the 480 image pixel by pixel using image calculator/divide function and the image was converted to 32-bit. Single optical sections in the middle of the eye were used to calculate average 405/480 ratio values in the retina. The region of interest (ROI) was defined by free-hand tool. Data were normalized to ratios before applying any oxidizing/reducing agents or control. For comparing basal levels in WT and nox2 mutant fish embryos, ratio values were normalized to WT fish. Data presented as mean \pm SEM. Paired and unpaired Student's $t$ test were performed to compare effects of $\mathrm{H}_{2} \mathrm{O}_{2}$ / DTT and the difference between WT and mutant, respectively. For ratio image display, a binarized mask was created first in ImageJ, in which ROI pixels (embryo) received a value of 1 , whereas background pixels were set to 0 . The binarized mask was then multiplied with the ratio image and a factor of 2 . The resulting ratiometric image was converted to 8-bit and pseudocolored by applying lookup table "Fire." The quantification of $\mathrm{H}_{2} \mathrm{O}_{2}$ levels and the creation of ratio- 
metric images of growth cones were done essentially the same way as for the embryos with the exception that a factor of 4 was applied when creating ratiometric images because intensities were lower in the growth cones than in whole embryos. Wilcoxon and Mann-Whitney tests were used to analyze growth cone $\mathrm{H}_{2} \mathrm{O}_{2}$ levels because data were not distributed normally.

Optokinetic response $(O K R)$. We used a custom-built apparatus to assess OKR in 5-d-old zebrafish embryos. The apparatus consists of a plastic drum with a lined paper made up of alternating black and white stripes, a stereoscope with a light source, and a variable speed motor and attached belt used to rotate the drum. Briefly, in each session, 5 larvae were embedded ventrally in a 3\% methyl-cellulose solution in a $35 \mathrm{~mm}$ plastic dish. The dish was placed in the middle of the rotating drum. Each larva was screened for $1 \mathrm{~min}$ with a series of rotations lasting $15 \mathrm{~s}$ and alternating between clockwise and counterclockwise direction turning of the drum at $10 \mathrm{rpm}$. Each larva was observed individually to assess the response to the rotating drum. The larva that followed the rotating stripes and adapts changes in the direction of the turning drum were classified as OKR responsive, whereas the larva that did not follow the stripes and exhibited no response at all to the rotating drum were classified as OKR nonresponsive. The test was repeated four-times independently and at least 10 fish were observed from each genotype per session.

Statistical analysis. Datasets were analyzed using GraphPad Prism software version 6.05. All raw data were assessed for normality using the D'Agostino-Pearson omnibus test included in GraphPad. All data displayed a normal distribution $(p \geq 0.05)$ and no transformations were necessary. Outliers were identified automatically and removed using the ROUT algorithm with a Q-value of $1 \%$. In all cases, $\leq 2$ outliers were identified per group. Because all datasets contained three or more groups, one-way ANOVA was used to identify differences among means and $p$-values $<0.05$ were considered significant. Corresponding $F$ statistics and $p$-values are reported in the Results section. If a significant difference was detected with the ANOVA, then Tukey's HSD method was used for multiple comparisons among groups or the Dunnett's test was used for specific comparisons to a control unless noted otherwise. The test used is specified in the figure legends. Tukey's or Dunnett's adjusted $p$-values are displayed in the figures and the Results section. Graphs in Figures $1,2,3,4$, and 5 show mean \pm SD for each group and graphs in Figure 6 show mean \pm SE or the mean. Values reported in the text reflect the information in the corresponding graph.

\section{Results}

\section{Nox inhibition leads to defects in retinotectal development}

To study the role of Nox function in nervous system development, we applied a pan-Nox inhibitor, celastrol (Jaquet et al., 2011), to zebrafish embryos and screened for neuroanatomical defects. This compound was chosen because it shows no antioxidant effects common in other Nox inhibitors such as apocynin (Heumüller et al., 2008). We observed defects specifically in the formation of the GCL and the ON when celastrol was applied between 32 and $36 \mathrm{hpf}$ (Fig. 1), as well as the formation of the anterior commissures (ACs) in the forebrain when the inhibitor was applied between 20 and $24 \mathrm{hpf}$ (data not shown). Because the zebrafish visual system is well characterized anatomically, functionally, and developmentally, we chose to focus this study on the development of the RGCs and ON. In zebrafish, the retina begins to differentiate at $28 \mathrm{hpf}$. At this time, RGCs differentiate in the GCL medial to the lens of the eye (Hu and Easter, 1999). At a point in the middle of the eye called the optic disk, axons throughout the GCL fasciculate into a single bundle called the ON. The left and right ONs then grow toward the midline of the embryo, where they cross at a point called the optic chiasm at 〜36 hpf (Burrill and Easter, 1995; Stenkamp, 2015). From the chiasm, ON axons grow dorsocaudally toward a midbrain region called the OT, where they form synapses with tectal neurons by 48 hpf (Kita et al., 2015a).
To assess the effects of Nox inhibition on retinal, ON, and OT development, celastrol was applied at $2.5 \mu \mathrm{M}$ during the period of ON fasciculation and growth toward the chiasm (32-36 hpf). The inhibitor was then washed out and the embryos were allowed to develop until ON axons reached the OT (48 hpf). Embryos were then fixed and immunostained with zn-8, an antibody that labels the GCL and ON (Avanesov and Malicki, 2010). We observed an expansion of the zn-8-positive GCL and a thinning of the ON in celastrol-treated fish embryos (Fig. $1 A, B$ ). We quantified GCL width and $\mathrm{ON}$ thickness as measures of retinal development (Fig. $1 D, E)$. Differences in GCL width were found to be statistically significant $\left(F_{(2,35)}=66.6, p<0.0001\right)$. Control GCLs had an average width of $100 \pm 10.6 \mu \mathrm{m}$ (mean $\pm \mathrm{SD}$; Fig. $1 A, D$ ). Inhibitor-treated embryos displayed a significant increase in GCL thickness compared with control $(128 \pm 5.0 \mu \mathrm{m}, p<$ 0.0001 ; Fig. $1 B, D$ ). Previous studies have shown that Nox loss of function can be rescued via the addition of $\mathrm{H}_{2} \mathrm{O}_{2}$ (Le Belle et al., 2011; Rieger and Sagasti, 2011). Therefore, we applied $2 \mathrm{~mm}$ $\mathrm{H}_{2} \mathrm{O}_{2}$ immediately after celastrol treatment; however, this did not affect the width of the GCL compared with celastrol-only-treated embryos $(127 \pm 3.6 \mu \mathrm{m}, p=0.7886$; Fig. $1 C, D)$. Differences among mean $\mathrm{ON}$ thickness were also found to be significant $\left(F_{(2,35)}=133.5, p<0.0001\right)$. Control embryos treated with $0.01 \%$ DMSO displayed a mean ON thickness of $25 \pm 3.4 \mu \mathrm{m}$ (Fig. $1 A, E$ ). ONs of celastrol-treated embryos were significantly thinner $(9 \pm 2.2 \mu \mathrm{m}, p<0.0001$ vs control; Fig. $1 B, E)$. Here, $\mathrm{H}_{2} \mathrm{O}_{2}$ application caused a marked recovery of $\mathrm{ON}$ thinning (17.9 $\pm 1.5 \mu \mathrm{m}, p<0.0001$ vs celastrol alone; Fig. $1 C, E)$.

We then used the $\operatorname{Tg}($ ath $5: G F P)$ transgenic line to label RGCs and the $\mathrm{ON}$ in live embryos. Using this strategy, we were able to image retinal development and OT innervation at different time points in the same embryo. In response to the Nox inhibitor treatment, the GCL again appeared wider (Fig. 1G). To determine whether this wider GCL represented an expansion of RGCs within the retina, we calculated the ratio of the GFP-positive area divided by the total area of the retina and found significant differences among groups $\left(F_{(2,47)}=159, p<0.0001\right)$. The GCL in inhibitor-treated embryos occupied a greater proportion of the retina versus controls, suggesting an increased number of RGCs in inhibitor-treated embryos (Fig. $1 L ; 0.45 \pm 0.05,0.24 \pm 0.03$, respectively, $p<0.0001$ ). Similar to our findings with $\mathrm{zn}-8$ labeling (Fig. $1 D$ ), treatment with $\mathrm{H}_{2} \mathrm{O}_{2}$ did not diminish significantly the ratio of the retina occupied by GFP-positive RGCs versus celastrol treatment $(0.47 \pm 0.04, p=0.43)$. We again found a significant difference among mean $\mathrm{ON}$ thickness in the three treatment groups $\left(F_{(2,47)}=85.6, p<0.0001\right)$. Control embryos had ONs with a mean thickness of $25.5 \pm 3.4 \mu \mathrm{m}$ (Fig. $1 F, M$ ), whereas ONs of inhibitor-treated embryos were significantly thinner compared with controls $(9.9 \pm 2.0 \mu \mathrm{m}, p<0.0001$; Fig. $1 G, M)$. The addition of $\mathrm{H}_{2} \mathrm{O}_{2}$ partially rescued $\mathrm{ON}$ thickness compared with celastrol treatment $(22.0 \pm 4.6 \mu \mathrm{m}, p<0.0001$; Fig. $1 H, M)$.

We next investigated the innervation of the OT in response to Nox inhibition. No differences in axon targeting within the OT were observed because all GFP signals localized to the presumptive OT, as determined by visual comparison with control embryos. However, we measured the area of the OT innervated by GFP-positive axons and found significant differences among groups $\left(F_{(2,53)}=105.3, p<0.0001\right)$. Controls displayed a mean area of $7998 \pm 1154 \mu \mathrm{m}^{2}$ at $72 \mathrm{hpf}($ Fig. $1 I, N)$. Celastrol-treated embryos showed a significant decrease in OT innervation $\left(3164 \pm 721 \mu \mathrm{m}^{2}, p<0.0001\right.$ vs control; Fig. $\left.1 \mathrm{~J}, N\right)$. Like ON thickness, OT innervation was partially rescued by the $2 \mathrm{~mm}$ 

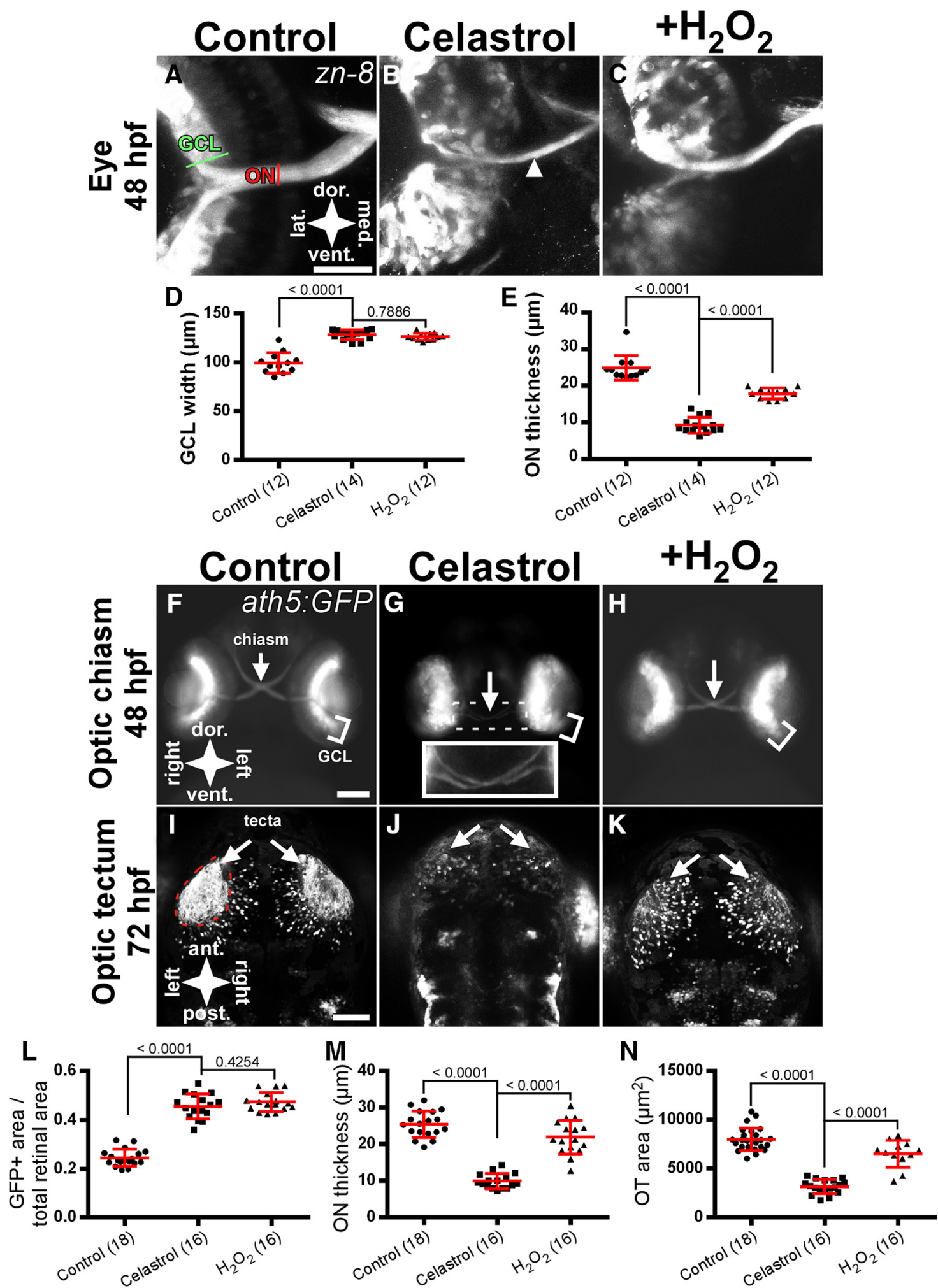

Figure 1. Nox inhibition causes defects in retinal development and tectal innervation. $\boldsymbol{A}-\boldsymbol{C}$, Retinal micrographs of $48 \mathrm{hpf}$ embryos labeled with the RGC marker $\mathrm{zn}-8$ after a $4 \mathrm{~h}$ treatment with $0.1 \%$ DMSO $(\boldsymbol{A})$ or $2.5 \mu \mathrm{m}$ celastrol ( $\boldsymbol{B}, \boldsymbol{C}$. Embryo in $\boldsymbol{C}$ was incubated with $2 \mathrm{~mm} \mathrm{H}_{2} \mathrm{O}_{2}$ after drug washout. White arrowhead in $\boldsymbol{B}$ indicates $0 \mathrm{~N}$ thinning. $\boldsymbol{D}$, Graph of GCL width in zn-8-labeled embryos treated with celastrol. $\boldsymbol{E}$, Graph of $0 \mathrm{~N}$ thickness in zn-8-labeled embryos treated with celastrol. $\boldsymbol{F}$ - $\boldsymbol{H}$, Anterior views of $48 \mathrm{hpf}$ Tg(ath5:GFP) embryos after treatment with DMSO (F) or celastrol with $(\boldsymbol{H})$ or without $(\boldsymbol{G})$ subsequent $\mathrm{H}_{2} \mathrm{O}_{2}$ incubation. Inset in $\boldsymbol{G}$ shows the optic chiasm. $\boldsymbol{I}-\boldsymbol{K}$, Dorsal views of control $(\boldsymbol{I})$, inhibitor-treated $(\boldsymbol{J})$, and $\mathrm{H}_{2} \mathrm{O}_{2}$ rescue $(\boldsymbol{K})$ embryos in 72 hpf embryos showing optic tectum innervation. White arrows indicate the optic chiasm $(\boldsymbol{F}-\boldsymbol{H})$ or optic tecta $(\boldsymbol{I}-\boldsymbol{K})$. $\boldsymbol{L}$, Graph of GFP-positive area corresponding to the GCL divided by total retinal area in Tg(ath5:GFP) embryos treated with celastrol. $\boldsymbol{M}$, Graph of $0 \mathrm{~N}$ thickness in $T g($ ath5:GFP) embryos treated with celastrol. $\boldsymbol{N}$, Graph of $0 \mathrm{~T}$ area in $T g($ ath5:GFP) embryos treated with celastrol. Scale bars, $50 \mu \mathrm{m}$ ( $\boldsymbol{A}-\boldsymbol{C}$ ) and $100 \mu \mathrm{m}(\boldsymbol{F}-\boldsymbol{K})$. All graphs show mean \pm SD. Numbers in parentheses indicate the number of embryos analyzed. $p$-values from Tukey's HSD tests are reported above comparison bars. 
A Nox1

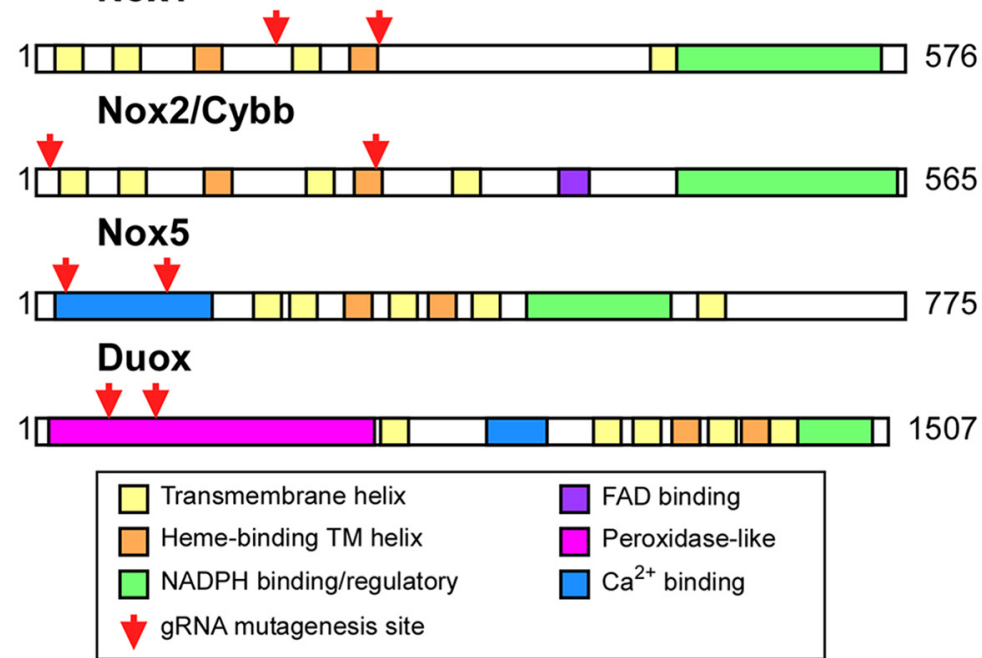

L
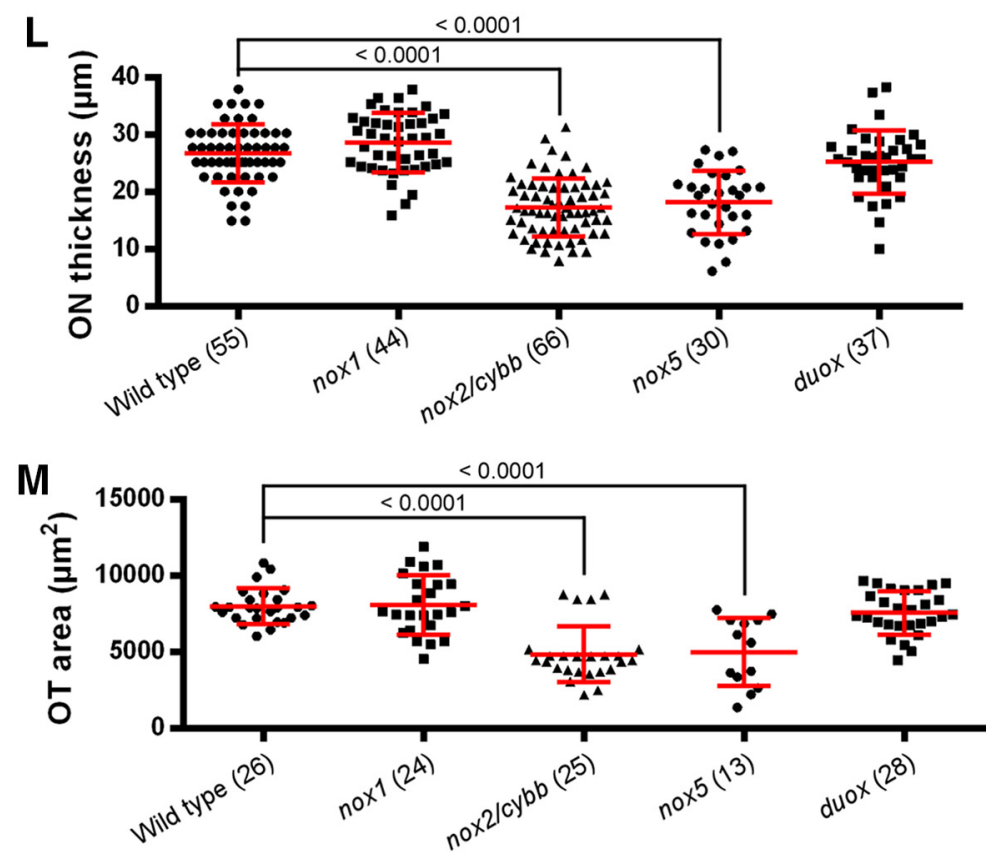

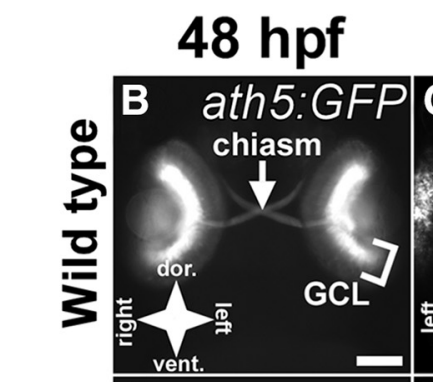

$72 \mathrm{hpf}$
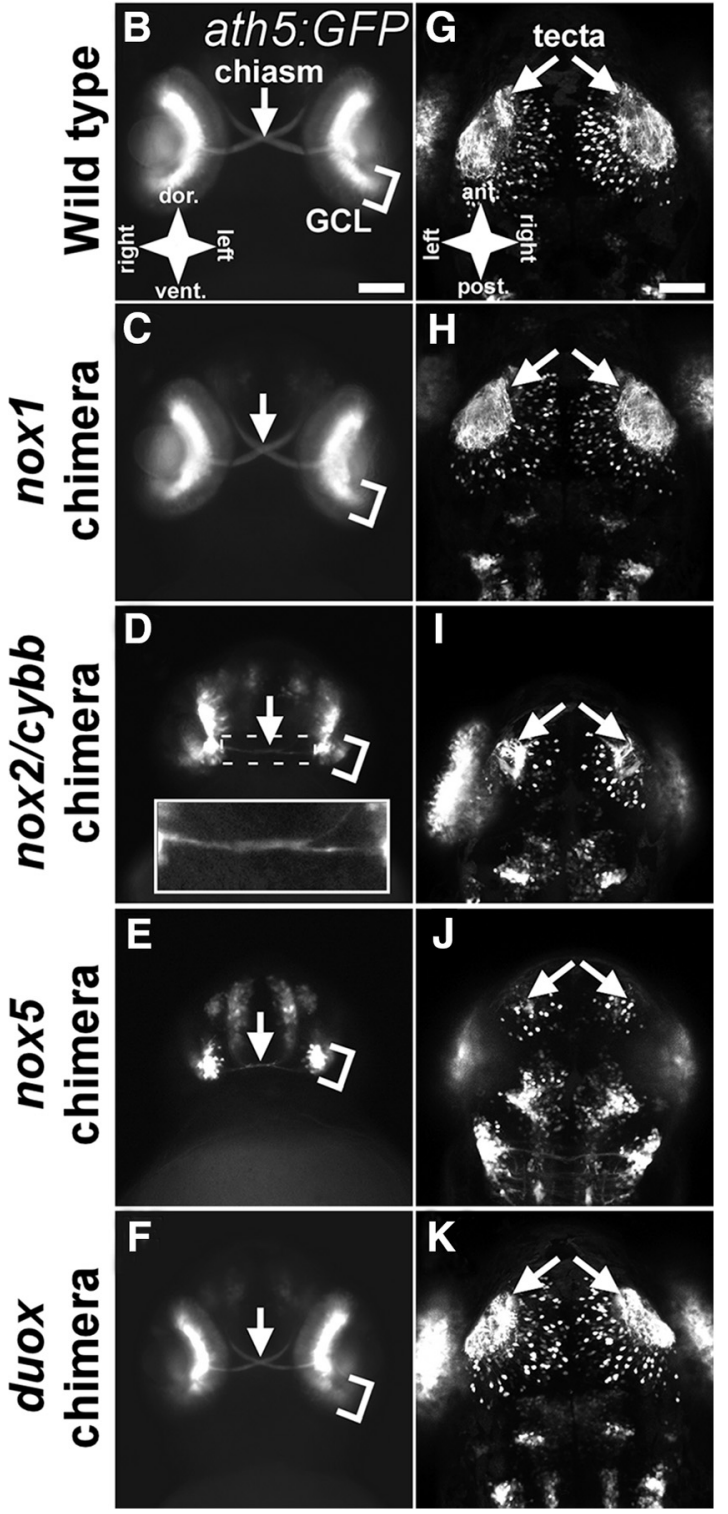

Figure 2. Chimeric nox mutants show optic nerve and tectal defects. $\boldsymbol{A}$, Schematic of zebrafish NOX protein domains as determined by alignment to the human protein sequences. Red arrows indicate the relative location of gRNA target sites. $\boldsymbol{B}-\boldsymbol{F}$, Anterior views $\mathrm{Tg}$ (ath5:GFP)-positive, nox chimeras at 48 hpf. White arrows indicate the optic chiasm. Inset in $\boldsymbol{D}$ shows in enlarged and enhanced view of the optic chiasm. $\mathbf{G}-\boldsymbol{K}$, Dorsal views of chimeric embryos showing the innervation of the optic tecta (white arrows). GFP-positive cells not indicated with arrows represent nontectal early neurons from the hindbrain and eye. Scale bars: $\boldsymbol{B}-\boldsymbol{F}, 100 \mu \mathrm{m} ; \boldsymbol{G}-\boldsymbol{K}, 50 \mu \mathrm{m}$ ). $\boldsymbol{L}$, Graph of ON thickness in $\operatorname{Tg}($ ath 5:GFP) positive, chimeric mutants. $\boldsymbol{M}, \mathbf{G r a p h}$ of $0 \mathrm{~T}$ area in $T g($ ath5:GFP)-positive, chimeric mutants. Numbers in parentheses indicate the number of embryos analyzed. Graphs show mean \pm SD. $p$-values from Dunnett's tests are reported above comparison bars.

$\mathrm{H}_{2} \mathrm{O}_{2}$ applied immediately after inhibitor treatment $(6538 \pm$ $1384 \mu \mathrm{m}^{2}, p<0.0001$ vs celastrol; Fig. $1 K-N$ ). Collectively, these results demonstrate that pharmacological Nox inhibition results in GCL expansion, ON thinning, and reduced OT innervation, indicating a critical role for NADPH oxidase in normal retinotectal development.

nox $2 / c y b b$ chimeric mutants display retinotectal defects To determine which specific Nox isoform is involved, we used the CRISPR/Cas9 genome editing technique to generate Nox isoform-specific mutations in zebrafish embryos. For improved validation, we designed two gRNAs per Nox isoform and injected them into one-cell-stage embryos to generate site-specific mutations in the nox genes. The locations of the gRNA target sites with respect to full-length Nox proteins are shown in Figure $2 A$. We
Table 4. Efficiency of gRNAs

\begin{tabular}{llllllll}
\hline & \multicolumn{3}{c}{ gRNAI } & & & \multicolumn{3}{c}{ gRNA II } \\
\cline { 2 - 3 } & Mutant & Total & $\%$ & & Mutant & Total & $\%$ \\
\hline nox1 & 50 & 55 & 91 & & 46 & 61 & 75 \\
nox2/cybb & 40 & 60 & 67 & & 49 & 58 & 84 \\
nox5 & 35 & 52 & 67 & 50 & 55 & 91 \\
duox & 49 & 55 & 89 & & 42 & 50 & 84 \\
\hline
\end{tabular}

Embryos were genotyped as described in the Materials and Methods. "Total" refers to the total number of embryos that were injected and genotyped; "mutant" refers to the number of embryos found to carry mutations.

first calculated the efficiency of the gRNAs by quantifying the percentage of mutant embryos generated by each gRNA (Table 4). All of the gRNAs produced mutations in at least $65 \%$ of the injected embryos. Some (e.g., nox1 gRNA I and nox5 gRNA II) 
generated mutations in $>90 \%$ of the injected embryos. We also genotyped the predicted off-target sites, shown in Table 2, for each gRNA and found no mutations at these locations in the genome (data not shown).

Using this validated set of Nox isoform-specific gRNAs, we investigated which Nox isoforms contribute to retinal development. We coinjected both gRNAs against a given nox gene into $\operatorname{Tg}$ (ath5:GFP) embryos, which generated a set of novel mutations in each embryo. Due to the random incorporation of insertions and deletions in the early cells, these embryos are chimeric. Therefore, these chimeric embryos retain an unknown percentage of WT nox genes in certain tissues. The implications of this will be addressed in the Discussion. The injected embryos were genotyped at both gRNA loci and the resulting phenotypes were assessed only in individuals carrying mutations at both gRNA sites. Restricting the analysis to double-site mutants maximizes the probability of producing a loss-of-function mutation. At least 20 chimeric mutants were analyzed for each gene and representative ON and OT images are shown in Figure 2, along with measurements of ON thickness at $48 \mathrm{hpf}$ and OT area at $72 \mathrm{hpf}$. Injected embryos that did not carry mutations at either gRNA site served as WT controls for these experiments (Fig. $2 B, G$ ). None of the nox chimeric mutants showed expansion of the GCL as in the case of inhibitor-treated embryos (Fig. $2 B-F$ ) or significant mistargeting of GFP-positive axons within the midbrain (Fig. $2 G-$ $K)$. Importantly, ON thickness and OT innervation in controls were similar to what was found previously in DMSO-treated embryos (26.8 $\pm 5.0 \mu \mathrm{m}$ and $7998 \pm 1154 \mu \mathrm{m}^{2}$, respectively; Fig. $2 B, G, L, M)$. ONs of nox 1 mutants were not significantly different from WT controls $(28.7 \pm 5.2 \mu \mathrm{m}, p=0.24$; Fig. $2 C, L)$. OT innervation was similarly unaffected in these mutants $(8063 \pm$ $1946 \mu \mathrm{m}, p=1.00$; Fig. $2 H, M)$. Similar to what was observed in response to pharmacological Nox inhibition, nox $2 / c y b b$ mutants displayed thin ONs $(17.4 \pm 5.1 \mu \mathrm{m}, p<0.0001$ vs control; Fig. $2 D, L)$. The OT of nox $2 / c y b b$ mutant larvae showed sparse GFP signals, indicating a reduced number of $\mathrm{ON}$ axons innervating the tissue ( $4840 \pm 1835 \mu \mathrm{m}^{2}, p<0.0001$ vs control; Fig. $\left.2 I, M\right)$. nox5 mutants also displayed ON thinning $(18.2 \pm 5.5 \mu \mathrm{m}, p<$ 0.0001 vs control; Fig. $2 E, L)$ and decreased tectal innervation (4995 $\pm 2243 \mu \mathrm{m}^{2}, p<0.0001$ vs control; Fig. $2 \mathrm{~J}, M$ ). However, nox5 mutants had severe general morphological defects early in development that might interfere with the formation of the brain and retina (data not shown). This is further evidenced by the fact that developing homozygous nox $5^{-1-}$ loss-of-function mutants has been quite challenging because many embryos do not develop fully. Therefore, we chose to focus on nox $2 / c y b b$ in subsequent experiments. Finally, duox mutants displayed insignificant $\mathrm{ON}$ thinning $(25.3 \pm 5.5 \mu \mathrm{m}, p=0.44$ vs control; Fig. $2 F, L)$ and decreased tectal innervation $\left(7556 \pm 1417 \mu \mathrm{m}^{2}, p=0.74\right.$ vs control; Fig. $2 K, M)$. In summary, these results showed that, among the different nox isoforms, nox $2 / c y b b$ plays a critical the development of the zebrafish visual system.

Tectal innervation is reduced in chimeric nox $2 / c y b b$ mutants The effect of nox $2 / c y b b$ mutation is a thin, underdeveloped $\mathrm{ON}$ at $48 \mathrm{hpf}$, the axons of which fail to properly innervate the OT by 72 hpf (Fig. 2D,I). To address whether these defects in retinotectal connectivity persist at later time points, we leveraged the liveimaging capability of the $\operatorname{Tg}($ ath $5: G F P)$ line to capture $\mathrm{ON}$ and OT images from nox $2 / c y b b$ chimeric embryos at multiple time points (Fig. 3). We also included a set of nox2/cybb chimeric mutants that were incubated with $2 \mathrm{mM} \mathrm{H}_{2} \mathrm{O}_{2}$ as a rescue control condition. As shown in Figure 3, $B$ and $C$, we observed charac- teristic ON thinning in nox $2 / c y b b$ mutants at $48 \mathrm{hpf}(14.7 \pm 3.7$ $\mu \mathrm{m}, p<0.0001$ vs control; Fig. $3 I$ ). ONs from nox $2 / c y b b$ mutants incubated with $\mathrm{H}_{2} \mathrm{O}_{2}$ were significantly thicker than untreated mutants $(19.8 \pm 3.4 \mu \mathrm{m}, p=0.0035$ vs mutant; Fig. $3 D, I)$, indicating that the phenotype observed in nox $2 / c y b b$ mutants is likely due to reduced $\mathrm{H}_{2} \mathrm{O}_{2}$ levels. We then imaged the same embryos at $72 \mathrm{hpf}$ to assess the persistence of $\mathrm{ON}$ thinning. By this time point, nox $2 / c y b b$ mutants recovered $(35.7 \pm 7.8 \mu \mathrm{m}$; Fig. $\left.3 B^{\prime}-C^{\prime}\right)$ such that they were nearly identical to controls $\left(39.9 \pm 6.9 \mu \mathrm{m}\right.$; Fig. $\left.3 A^{\prime}\right) . \mathrm{H}_{2} \mathrm{O}_{2}$-treated larvae were similar to controls with a thickness of $37.3 \pm 5.2 \mu \mathrm{m}$ (Fig. $3 D^{\prime}$ ). No significant differences were detected among groups, suggesting that the $\mathrm{ON}$ phenotypes observed in response to nox $2 / c y b b$ mutation could be transient and likely due to developmental delays $\left(F_{(2,57)}=2.086, p=0.7842\right)$.

We then turned our attention to the effects of nox $2 / c y b b \mathrm{mu}$ tations on OT innervation. Imaging the same samples over $3 \mathrm{~d}$, we saw a progressive increase in tectal innervation between 72 and $96 \mathrm{hpf}$ in WT controls (Fig. $3 E-H, E^{\prime}-H^{\prime}, J$ ). Significant differences among groups were identified at both time points $\left(F_{(2,48)}=38.37, p<0.0001\right.$ for $72 \mathrm{hpf} ; F_{(2,48)}=9.106, p=0.0004$ for $96 \mathrm{hpf}$ ). Innervated areas in the OT from nox $2 / c y b b$ mutants were significantly smaller than control $(p<0.0001)$ and $\mathrm{H}_{2} \mathrm{O}_{2}$ rescue conditions $(p<0.0001)$ at $72 \mathrm{hpf}($ Fig. $3 E-H, J)$. By 96 hpf, innervated tectal areas of mutants and $\mathrm{H}_{2} \mathrm{O}_{2}$-treated mutants were still smaller than controls $(p=0.0006$ and 0.005 , respectively), but were not different from one another $(p=0.72$; Fig. $\left.3 E^{\prime}-H^{\prime}, J\right)$. A more severe loss of OT innervation was detected in $n o x 2 / c y b b$ mutants by $120 \mathrm{hpf}\left(F_{(2,48)}=291.9, p<0.0001\right)$. OT innervation in mutants drops to $3590 \pm 1357 \mu \mathrm{m}^{2}(p<0.0001$ vs control; Fig. $\left.3 F^{\prime \prime}, G^{\prime \prime}, J\right)$ compared with WT controls $\left(13,505 \pm 844 \mu \mathrm{m}^{2}\right.$; Fig. $\left.3 E^{\prime \prime}, J\right)$ and is significantly different from $\mathrm{H}_{2} \mathrm{O}_{2}$-treated mutants $\left(11,462 \pm 1444 \mu \mathrm{m}^{2}, p<0.0001\right.$; Fig. $\left.3 H^{\prime \prime}, J\right)$. These results suggest that innervation of the optic tectum is reduced in $n o x 2 / c y b b$ chimeras.

\section{nox $2 / c y b b^{-1-}$ embryos show expansion of the GCL}

To minimize the potential phenotypic variability of nox $2 / c y b b$ mutant chimeras, we generated stable fish lines harboring homozygous nox $2 / c y b b$ mutations at either site targeted by the $n o x 2 / c y b b$ gRNAs (referred to as nox $2 / c y b b^{-1-}$ ). Two separate lines carrying mutations at different loci within the nox $2 / c y b b$ gene were generated to confirm the convergence of phenotypes and to ensure the specificity of mutagenesis. The mutant line generated from gRNA I had a 5 bp deletion, whereas the line from gRNA II carried a 23 bp deletion (Fig. 4A). Each line carries a premature stop codon early in the coding sequence for nox $2 / c y b b$, which results in a significant truncation of the Nox2/Cybb protein (Fig. 4B). Both Nox2/Cybb mutant enzyme types have lost the $\mathrm{C}$-terminal substrate binding domain. Four histidine residues within $\alpha$ helices III and V are essential for electron shuttling to the $\mathrm{O}_{2}$ acceptor and at least one of these critical histidines are missing in both mutant types (Kawahara and Lambeth, 2007; Brandes et al., 2014). Without these critical functional domains, the mutant Nox2/Cybb proteins are likely not functional.

Because $n o x 2 / c y b b^{-1-}$ mutant lines do not carry the $\mathrm{Tg}$ (ath5: GFP) transgene, we used zn-8 antibodies to visualize axons. Samples were also counterstained with the nuclear label DAPI to assess overall retinal development and lamination. In these experiments, WT clutch mates served as controls. In contrast to chimeras, nox $2 / c y b b^{-1-}$ embryos exhibited significant expansion of the zn-8-positive GCL at $48 \mathrm{hpf}\left(F_{(2,41)}=63, p<0.0001\right)$. The GCL in gRNA I $\left(0.49 \pm 0.06\right.$; Fig. $\left.4 D-D^{\prime \prime}, I\right)$ and gRNA II 

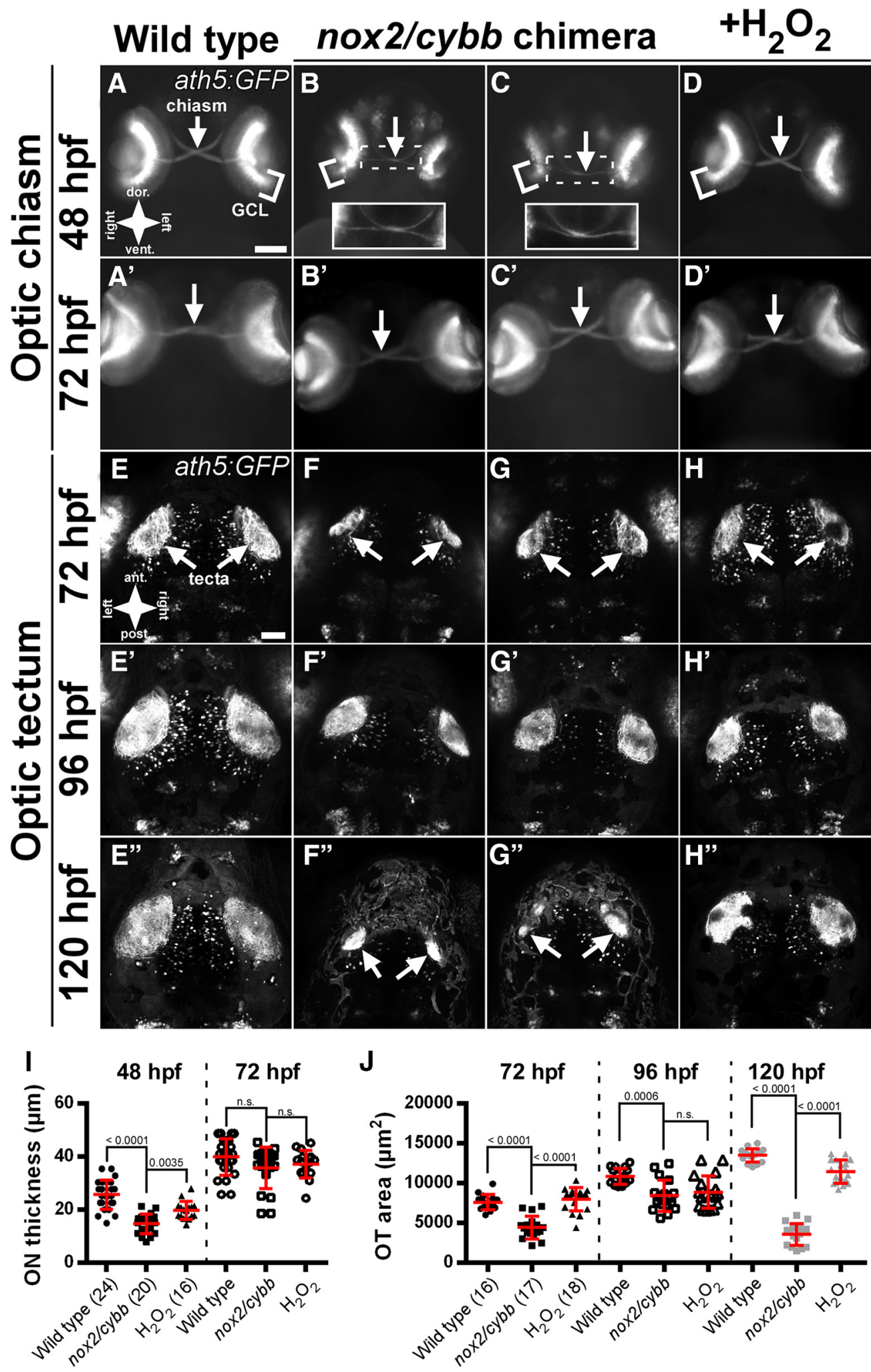

Figure 3. ON formation and OT innervation are impaired in chimeric nox2/cybb mutants. $A-D$, Anterior views of nox $2 / c y b b$ chimeras at $48 \mathrm{hpf}$ showing the formation of the optic chiasm. Insets in $\boldsymbol{B}$ and $\boldsymbol{C}$ show enlarged and enhanced views of the chiasm. $\boldsymbol{A}^{\prime}-\boldsymbol{D}^{\prime}$, Same samples as in $\boldsymbol{A}-\boldsymbol{D}$ imaged at $72 \mathrm{hpf}$ showing recovery of $0 \mathrm{~N}$ outgrowth in chimeras. $\boldsymbol{E}, \boldsymbol{F}$, Dorsal views of nox $2 /(\mathrm{cyb} b$ chimeras at $72 \mathrm{hpf} \mathrm{showing} \mathrm{the} \mathrm{innervation} \mathrm{of} \mathrm{the} \mathrm{optic} \mathrm{tecta.} \mathrm{The} \mathrm{same} \mathrm{samples} \mathrm{were} \mathrm{imaged} \mathrm{at} 96\left(\boldsymbol{E}^{\prime}-\boldsymbol{H}^{\prime}\right)$ and $120 \mathrm{hpf}\left(\boldsymbol{E}^{\prime \prime}-\boldsymbol{H}^{\prime \prime}\right)$ to assess the extent of recovery. White arrows indicate the optic chiasm $\left(\boldsymbol{A}-\boldsymbol{D}^{\prime}\right)$ or optic tecta $\left(\boldsymbol{E}-\boldsymbol{H}^{\prime \prime}\right)$. Scale bars: $\boldsymbol{A}-\boldsymbol{D}^{\prime}, 100 \mu \mathrm{m} ; \boldsymbol{E}-\boldsymbol{H}^{\prime \prime}, 50 \mu \mathrm{m}$. I, Graph of 0N thickness in $T g(a t h 5: G F P)$-positive, nox2/cybb chimeric mutants. $\boldsymbol{J}$, Graph of $0 \mathrm{~T}$ area in Tg(ath5:GFP)-positive, nox2/cybb chimeric mutants. Numbers in parentheses indicate the number of embryos analyzed. Graphs show mean \pm SD. $p$-values from Tukey's HSD tests are reported above comparison bars. n.s., No significant difference. 
A

nox 2/cybb gRNA I

Native nox $2 / c y b b$

Mutant nox $2 / c y b b$
TCATTTGCAGCAAAGTTTCC

TCCTTCATTTGCAGCAAAGTTTCCCATGATGT TCC ...........TTGCAGCAAAGTTTCCCATGATGT
B

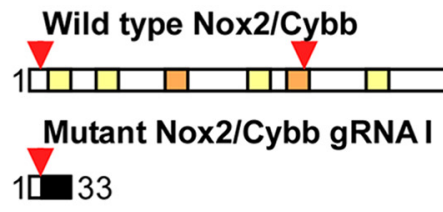

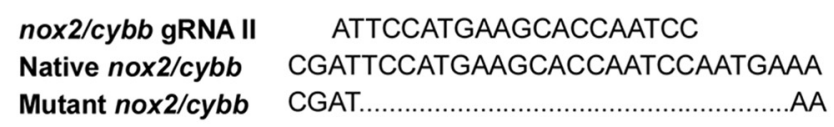

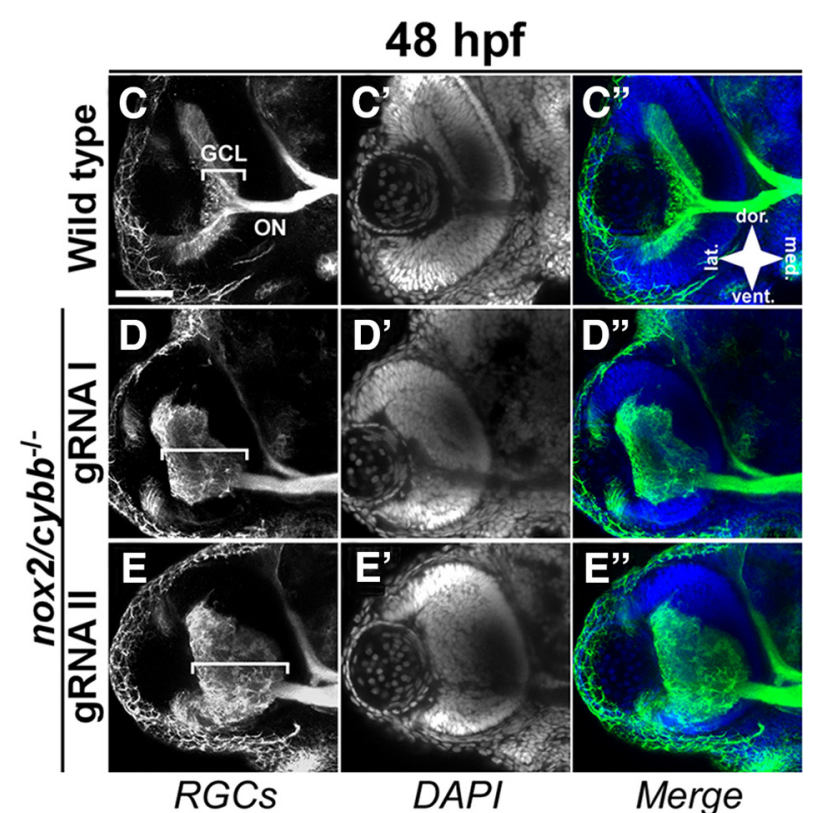

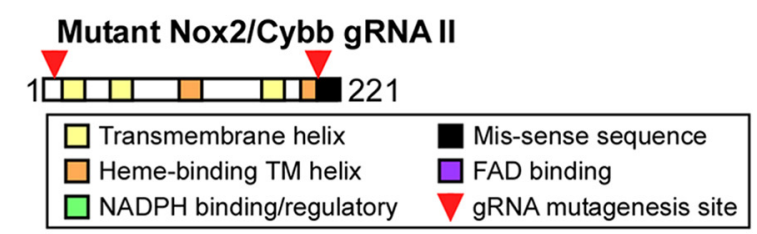

$72 \mathrm{hpf}$
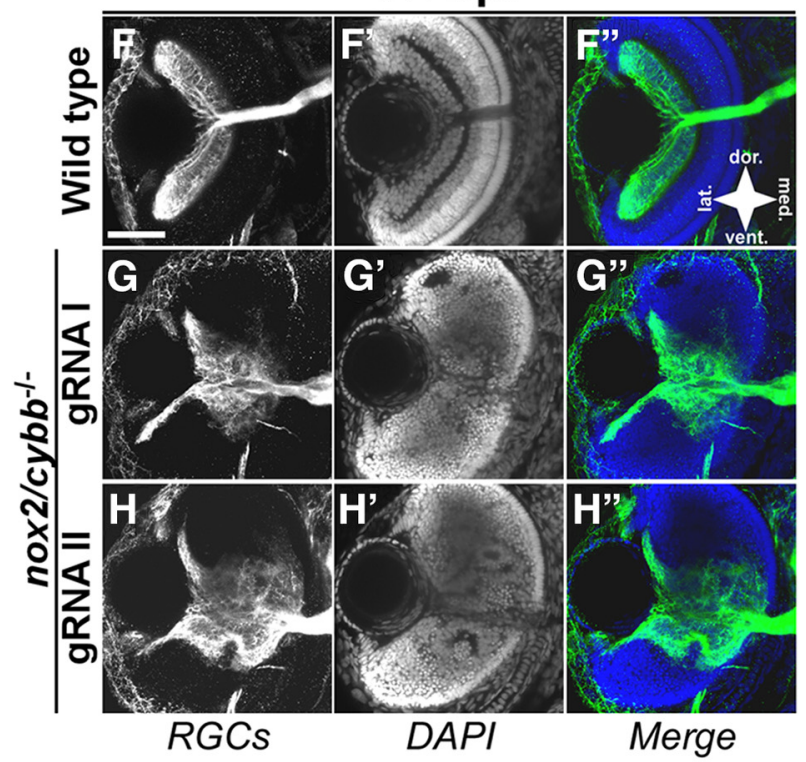

RGCS

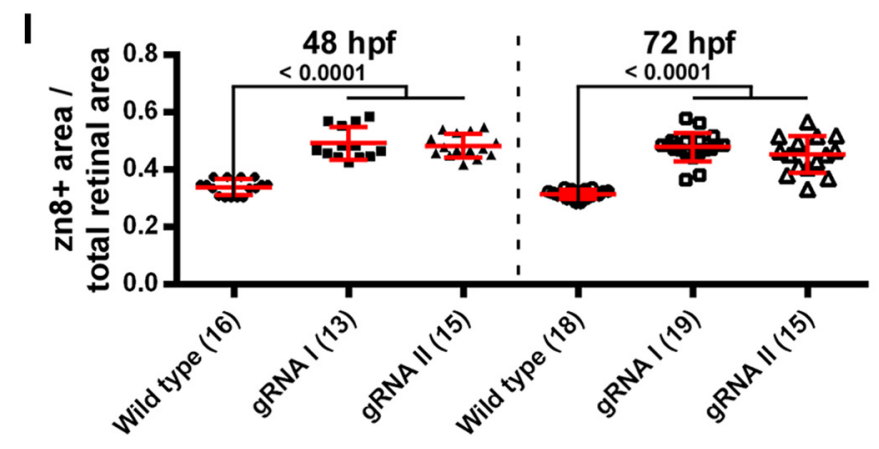

Figure 4. Homozygous nox2/cybb mutants exhibit GCL expansion. $A$, Genomic sequences of gRNA target sites along with nox2/cybb mutants showing the 5 and 23 bp deletions generated from gRNA I and II, respectively. $\boldsymbol{B}$, Schematic of the mutant Nox2/Cybb proteins based on translated mRNA sequences and alignment to the human protein sequence. $\boldsymbol{C}-\boldsymbol{E}^{\prime \prime}$, Micrographs of 48 hpf nox2/cybb $b^{-1-}$ eyes labeled with the RGC marker, zn-8 $(\boldsymbol{C}-\boldsymbol{E})$, and DAPI $\left(\boldsymbol{C}^{\prime}-\boldsymbol{E}^{\prime}\right)$. Mutants show defects in RGC outgrowth and retinal lamination. White brackets mark the ganglion cell layer. $\boldsymbol{F}-\boldsymbol{H}^{\prime \prime}{ }^{\prime}$ nox2/cybb ${ }^{-1-}$ eyes at $72 \mathrm{hpf} \mathrm{labeled} \mathrm{with} \mathrm{zn-8}(\boldsymbol{F}-\boldsymbol{H})$ and DAPI $\left(\boldsymbol{F}^{\prime}-\boldsymbol{H}^{\prime}\right)$ showing that the phenotype does not recover at later stages. Scale bars, $100 \mu \mathrm{m} . \boldsymbol{I}$, Graph of zn-8-positive area corresponding to the $\mathrm{GCL}$ divided by total retinal area in nox2/cybb ${ }^{-1-}$ mutants. Numbers in parentheses indicate the number of embryos analyzed. Graph shows mean $\pm \mathrm{SD}$. $p$-values from Dunnett's tests are reported above comparison bars.

$\left(0.48 \pm 0.04\right.$; Fig. $\left.4 E-E^{\prime \prime}, I\right)$ mutants occupies a greater proportion of the retina compared with controls $(0.34 \pm 0.03, p<$ 0.0001 vs mutants; Fig. $\left.4 C-C^{\prime \prime}, I\right)$. Unlike the $\mathrm{ON}$ phenotypes observed in chimeric mutants, GCL expansion did not recover by $72 \mathrm{hpf}$ in nox $2 / \mathrm{cybb} b^{-/-}$mutants and many cells were pyknotic at this later stage (Fig. $4 G^{\prime}-H^{\prime}$ ). In controls, the WT GCL occupied a similar percentage of the retina at $72 \mathrm{hpf}$ compared with that at 48 hpf $\left(0.31 \pm 0.02\right.$; Fig. $\left.4 F-F^{\prime \prime}, I\right)$ Mutant GCLs represented a significantly higher proportion of the retina compared with WT controls (gRNA I $0.48 \pm 0.05$, gRNA II $0.45 \pm 0.06, p<0.0001$; Fig. $\left.4 G-H^{\prime \prime}, I\right)$. These results further confirm a role for Nox2/
Cybb in GCL development. Interestingly, the ON was not significantly thinner in the homozygous mutants compared with WT controls (data not shown). The phenotypic differences observed among inhibitor-treated, chimeric, and homozygous mutant embryos will be addressed in the Discussion.

nox $2 / c y b b^{-1-}$ mutants exhibit mistargeted axons in the OT Next, we investigated the effects of homozygous nox $2 / c y b b^{-1-}$ loss-of-function mutations on OT innervation. The area of the midbrain occupied by innervating $\mathrm{ON}$ axons in WT embryos is largely devoid of DAPI-positive cell bodies (Fig. $5 A-A^{\prime \prime}$ ). This 
72 hpf

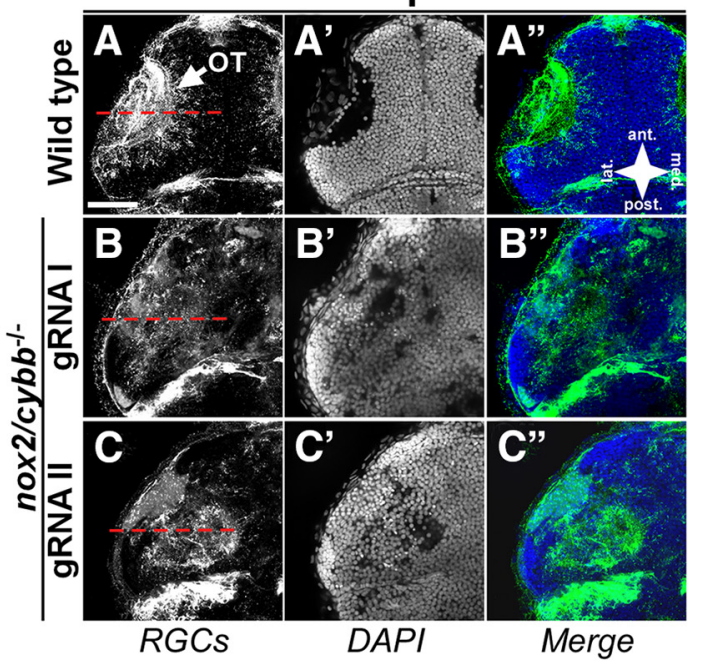

G

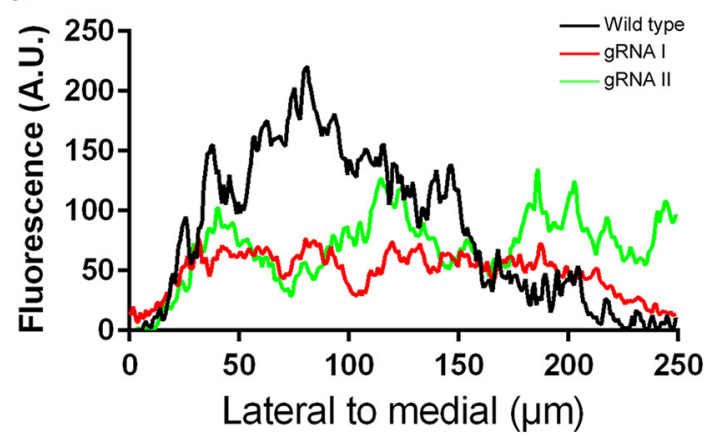

\section{$120 \mathrm{hpf}$}
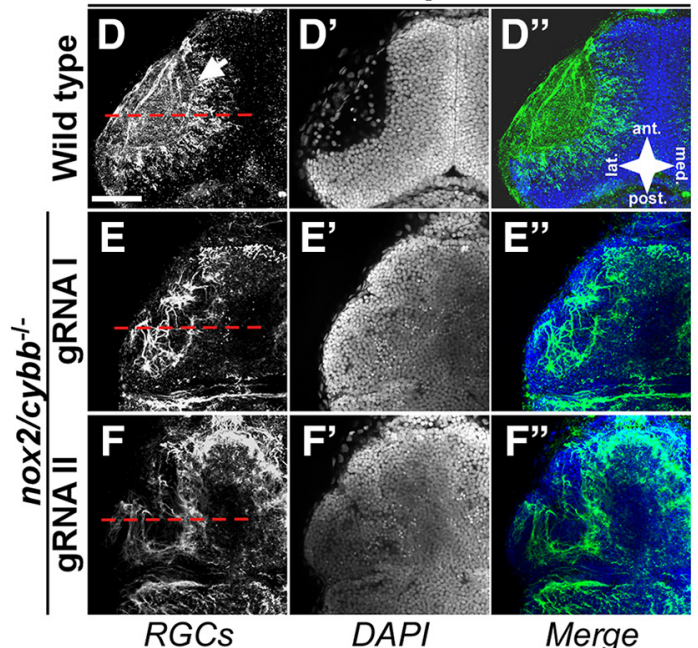

H

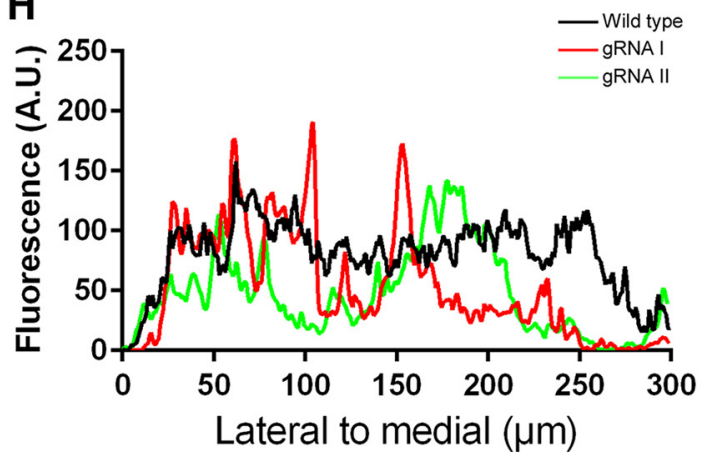

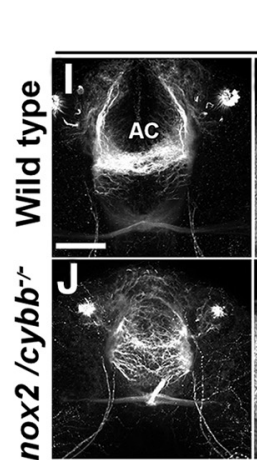

Acetylated Tubulin

\section{forebrain}
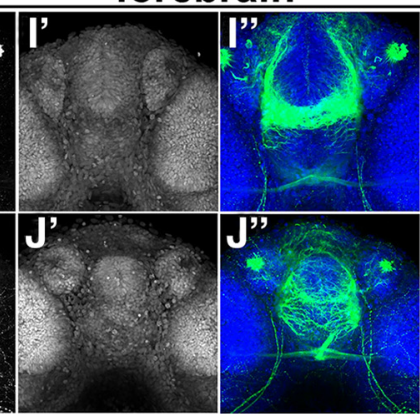

$D A P I$

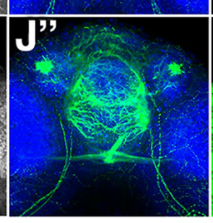

Merge

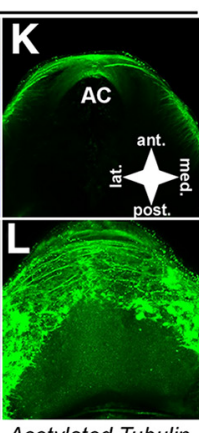

Acetylated Tubulin

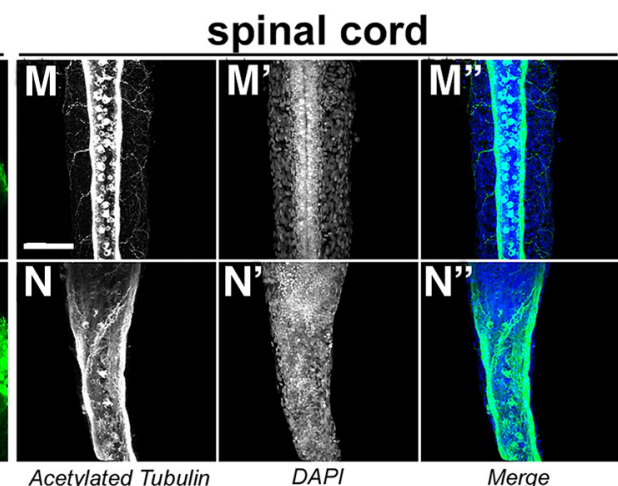

Figure 5. Axons are mistargeted in the 0 T of homozygous nox2/cybb mutants. $\boldsymbol{A}-\boldsymbol{C}^{\prime \prime}{ }^{\prime}$, Micrographs of $72 \mathrm{hpf} \mathrm{WT}(\boldsymbol{A})$ and nox2/cybb ${ }^{-/-}(\boldsymbol{B}, \boldsymbol{C})$ midbrains labeled with zn-8 $(\boldsymbol{A}-\boldsymbol{C})$ and DAPI $\left(\boldsymbol{A}^{\prime}-\boldsymbol{C}^{\prime}\right)$. Mutants show defects in tectal innervation. $\boldsymbol{D}-\boldsymbol{F}^{\prime \prime}$, WT $(\boldsymbol{D})$ and nox2/cybb ${ }^{-1-}$ midbrains at 120 hpf labeled with zn-8 $(\boldsymbol{E}, \boldsymbol{F})$ and DAPI $\left(\boldsymbol{E}^{\prime}-\boldsymbol{F}^{\prime}\right)$ showing that the phenotype does not recover at later stages. White arrows indicate the optic tecta. $G, \boldsymbol{H}$, Line scans plotting fluorescent intensity of zn-8 signals versus position in the midbrain. Placements of the line scans are shown with red dashed lines in zn-8 panels. $I-\boldsymbol{L}$, Micrographs of $48 \mathrm{hpf} \mathrm{WT}(\boldsymbol{I}-\boldsymbol{K})$ and nox2/cybb ${ }^{-I-}(\boldsymbol{J}-\boldsymbol{L})$ forebrains labeled with anti-acetylated $\alpha$-tubulin antibody and DAPI. nox2/cybb ${ }^{-/-}$embryos exhibit aberrant axonal projections at the forebrain AC. Ventral views are shown in I and $\boldsymbol{J}$; dorsal views in $\boldsymbol{K}$ and $\boldsymbol{L}$. $\boldsymbol{M}-\boldsymbol{N}^{\prime \prime}$, Micrographs of $48 \mathrm{hpf}$ WT $\left(\boldsymbol{M}-\boldsymbol{M}^{\prime \prime}\right)$ and nox2/cybb $b^{-I-}\left(\boldsymbol{N}-\boldsymbol{N}^{\prime \prime}\right)$ spinal cord labeled with anti-acetylated $\alpha$-tubulin antibody and DAPI. The spinal cord is wider and shows aberrant projections of the dorsal longitudinal fascicle in nox $2 /\left(\mathrm{cybb} b^{-1-}\right.$ embryos $\left(\boldsymbol{N}-\boldsymbol{N}^{\prime \prime}\right) \operatorname{compared}$ with WT embryos $\left(\boldsymbol{M}-\boldsymbol{M}^{\prime \prime}\right)$. Scale bars, $100 \mu \mathrm{m}$.

represents a prototypical morphology of the zebrafish OT (Kita et al., 2015a). In nox $2 / c y b b^{-1-}$ mutants, $z n-8$-positive axons are mistargeted to adjacent midbrain regions outside of the presumptive OT (Fig. $5 B, C$ ). In addition, there is no area devoid of DAPI-positive cell bodies in the presumptive location of the OT, suggesting the absence of a single nerve bundle entering the midbrain (Fig. $5 B^{\prime}-C^{\prime}$ ). To better evaluate the changes in tectal innervation, we used line scans visualizing the changes in labeling intensity across the tectum. In WT embryos at $72 \mathrm{hpf}$, fluores- cence intensity is highest in the area corresponding to the OT and falls precipitously in adjacent regions (Fig. $5 B-C^{\prime \prime}$ ). In contrast, line scans through tectal areas of nox $2 / c y b b^{-1-}$ mutants reveal lower signals in presumptive tectal areas, whereas $\mathrm{zn}-8$-positive axons can be found in extratectal areas (Fig. 5 B, C,G). By $120 \mathrm{hpf}$, a significant number of $\mathrm{zn}$-8-positive axons in the midbrain of nox $2 / c y b b^{-1-}$ mutants were not targeted uniformly to the presumptive OT (Fig. 5E,F). Again, DAPI staining in mutant embryos failed to show an area free of cell bodies indicative of an 
axon tract (Fig. $5 E^{\prime}, F^{\prime}$ ). Line scans continued to show a reduced and more diffuse zn-8 signals in tecta of mutants compared with WT embryos (Fig. $5 H$ ). Collectively, these results show that nox2/ $c y b b^{-1-}$ embryos exhibit defective $\mathrm{ON}$ development that results in improper axonal targeting within the OT. To determine whether no $\times 2 / c y b b^{-1-}$ embryos have impaired vision, we performed the OKR assay with 5-d-old embryonic zebrafish. Whereas all WT embryos responded to the rotating stripe pattern, as expected, all nox $2 / c y b b^{-1-}$ mutant embryos were scored as nonresponsive in the OKR assay (10 embryos in each group, four independent experiments), suggesting that mutant embryos are unable to detect motion.

\section{Abnormal axonal projections in other parts of the CNS of nox $2 / c y b b^{-/-}$mutants}

To determine whether axonal mistargeting in nox $2 / c y b b^{-1-}$ mutants is specific to the retinotectal pathway, we also investigated other areas of the CNS. Acetylated tubulin staining of axons in the forebrain showed aberrant axonal projections in $48 \mathrm{hpf}$ nox $2 /$ $c y b b^{-1-}$ mutant zebrafish embryos (Fig. 5I-L). In WT embryos, the AC in the telencephalon consists of fasciculated axons (Fig. $\left.5 I-I^{\prime \prime}\right)$. However, in nox $2 / c y b b^{-1-}$ mutants, the axons in the AC are not held together and the projections exhibit an irregular pattern (Fig. 5J- $J^{\prime \prime}$ ). The diminished fasciculation of axons in the AC can also been seen in the dorsal views (Fig. $5 K, L$ ) and is supported by our Nox inhibitor data (data not shown). Last, abnormal axonal projections were detected in the dorsal longitudinal fascicle of the spinal cord in $48 \mathrm{hpf} n o \times 2 / c y b b^{-1-}$ mutants (Fig. $5 M-N^{\prime \prime}$ ).

Because axonal tracts in different parts of the CNS are affected in nox $2 / c y b b^{-1-}$ mutants, we investigated whether the early development of the nervous system such as patterning is altered in these mutants to distinguish early developmental versus axonal guidance deficits. Therefore, we performed in situ hybridization experiments with known CNS gene markers for forebrain (pax6a), midbrain (ot $x 2$ ), and midbrain-hindbrain boundary ( $f g f 8)$ on $36 \mathrm{hpf}$ WT and nox $2 / c y b b^{-1-}$ mutant embryos (Fig. $\left.6 A-C^{\prime}\right)$. We did not find any obvious difference in the expression pattern of these marker genes between WT and nox $2 / c y b b^{-1-}$ mutant embryos. pax6a expression was found in the lens, retina, telencephalon, and hindbrain (Fig. $6 A, A^{\prime}$ ). otx2 expression was detected in the midbrain (Fig. $6 B, B^{\prime}$ ) and $f g f 8$ expression at the midbrain-hindbrain boundary (Fig. $6 C, C^{\prime}$ ). A recent study showed that both the number of neural progenitor cells and the level of nestin expression, a marker for neural progenitor cells, are decreased in nox2-deficient adult mice (Nayernia et al., 2017). We did not find any difference in nestin expression pattern between WT and mutant embryos at $36 \mathrm{hpf}$ (Fig. 6D, $D^{\prime}$ ). In summary, these data suggest that the major brain regions are intact and that the early development of CNS is not significantly altered in nox $2 / \mathrm{cybb}^{-1-}$ mutants. Overall, the results suggest that the axonal deficits in $n o x 2 / c y b b^{-1-}$ mutants are not due to general developmental problems and that Nox2 plays a role in axonal pathfinding and targeting.

\section{Nox inhibition reduces neurite outgrowth of cultured RGCs}

Our results so far show that lack of functional Nox2/Cybb leads to defects in GCL formation and tectal innervation. Next, we wanted to test whether Nox has a cell-autonomous role in neurite outgrowth of embryonic zebrafish RGCs. Because the in vivo results on Nox inhibition and Nox isoform mutants present a complex phenotype that may involve Nox function in multiple cell types, we turned instead to an in vitro culture system to study Nox function specifically in isolated RGC neurons (Fig. 7). Dissociated RGCs were harvested from $\operatorname{Tg}($ ath $5: G F P)$ embryos and plated on laminin-coated coverslips. Using cells derived from $\operatorname{Tg}$ (ath5:GFP) transgenic lines at $36 \mathrm{hpf}$, we were able to restrict our analysis to GFP-positive RGCs. In response to control treatments with $0.1 \%$ DMSO, RGCs exhibited average growth rates of $9.9 \pm 2.3 \mu \mathrm{m} / \mathrm{h}$ (mean \pm SEM; Fig. $7 A, A^{\prime}, C$ ), which is consistent with growth rates of cultured RGCs published by others (Chen et al., 2013) and with a recent study of axon outgrowth in vivo (Kita et al., 2015b). We treated cultured RGCs with various concentrations of celastrol to inhibit Nox. Significant differences in growth rates were identified $\left(F_{(4,173)}=3.3, p=0.0109\right)$. Neurite growth rates in celastrol-treated RGCs were decreased compared with controls and the effect of celastrol on neurite growth rate was dose dependent (Fig. 7C). The highest dose of celastrol, $0.5 \mu \mathrm{M}$, resulted in a significant decrease in growth rates $(0.5 \pm 2.5 \mu \mathrm{m} / \mathrm{h}$, $p=0.009$ vs control; Fig. $7 C)$. We confirmed these results using a second Nox inhibitor, VAS2870, and again saw differences among mean outgrowth rates $\left(F_{(3,274)}=4.6, p=0.0035\right)$. The highest dose of VAS2870, $1.0 \mu \mathrm{M}$, caused neurites to retract $(-2.0 \pm 3.5 \mu \mathrm{m} / \mathrm{h}, p=0.0019$ vs control; Fig. $7 D)$. These experiments show that RGC axons exhibit reduced growth when Nox enzymes are inhibited pharmacologically. Furthermore, we found that neurite growth rates of RGC neurons derived from nox $2 / c y b b^{-1-}$ mutants are reduced compared with WT neurons, although such reduction was not statistically significant (H.R. et al., unpublished data). This minor effect on neurite outgrowth in no $2 / \mathrm{cybb} b^{-1-}$ mutants could be due to the fact that specific molecular cues that activate Nox2 signaling and related axonal growth/guidance in vivo are missing in our cell culture system. In summary, our in vitro data indicate that at least some of the defects in the establishing retinotectal connections observed in no $2 / \mathrm{cybb} b^{-1-}$ mutants and Nox-inhibited embryos could be due to lack of functional Nox in RGC neurons, supporting a cellautonomous function of Nox in axonal growth and pathfinding of RGC axons.

\section{$\mathrm{H}_{2} \mathrm{O}_{2}$ levels in homozygous nox $2 / c y b b^{-/-}$mutants}

The results presented so far indicate that reducing Nox activity in general (through pharmacological Nox inhibition) or reducing Nox 2 activity in particular (in nox $2 / c y b b^{-1-}$ mutants) affects RGC differentiation and tectal innervation. To determine whether ROS levels are altered in nox $2 / c y b b^{-1-}$ mutants, we transiently expressed a genetically encoded $\mathrm{H}_{2} \mathrm{O}_{2}$-biosensor, roGFP2-orp1, in developing zebrafish embryos (Gutscher et al., 2009). Although superoxide is the primary ROS produced by Nox, superoxide can quickly react into $\mathrm{H}_{2} \mathrm{O}_{2}$, which has a longer half-life and therefore likely acts as the main ROS signaling molecule. First, we validated this $\mathrm{H}_{2} \mathrm{O}_{2}$ biosensor by treating $48 \mathrm{hpf}$ zebrafish embryos with $20 \mathrm{mM} \mathrm{H}_{2} \mathrm{O}_{2}$ for $10 \mathrm{~min}$, followed by 2.5 mM DTT for another $10 \mathrm{~min}$ while collecting ratiometric fluorescent images of the head region (Fig. 8A). We observed that $\mathrm{H}_{2} \mathrm{O}_{2}$ levels in the retina increased threefold with $\mathrm{H}_{2} \mathrm{O}_{2}$ treatment $(p<$ 0.0001 ; paired $t$ test), which was then reduced to $146 \%$ of the baseline level after the DTT treatment (Fig. $8 B$ ). Although we observed a slightly increased level of $\mathrm{H}_{2} \mathrm{O}_{2}$ in the mutant embryos, the trend was not significant ( $p=0.2675$; unpaired $t$ test with Welch's correction; Fig. $8 C$ ). We also assessed $\mathrm{H}_{2} \mathrm{O}_{2}$ levels in cultured RGC neurons at 34 hpf. Treatment of cultured RGC neurons with $100 \mu \mathrm{M} \mathrm{H} \mathrm{H}_{2} \mathrm{O}_{2}$ for 30 min increased growth cone $\mathrm{H}_{2} \mathrm{O}_{2}$ levels by $110 \%$ ( $p=0.0009$; Wilcoxon matched pairs test; 

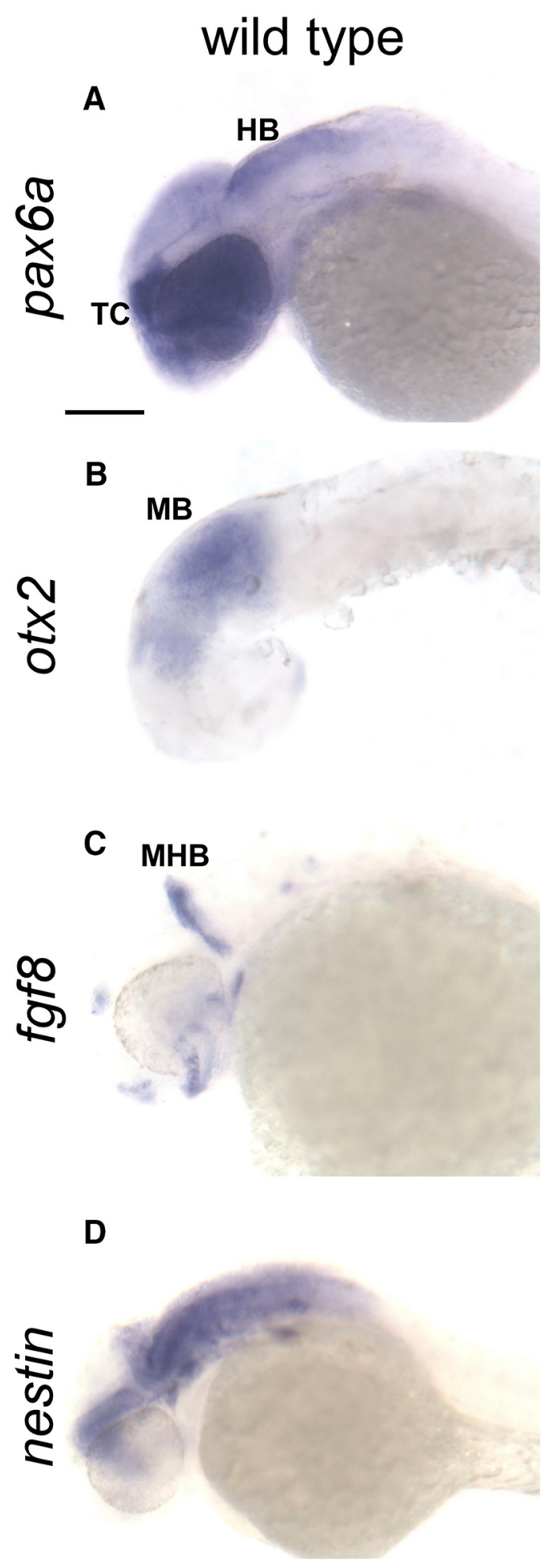

\section{$n o \times 2 / c y b b^{-/-}$}

$\mathbf{A}^{\prime}$

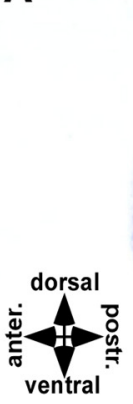

B'

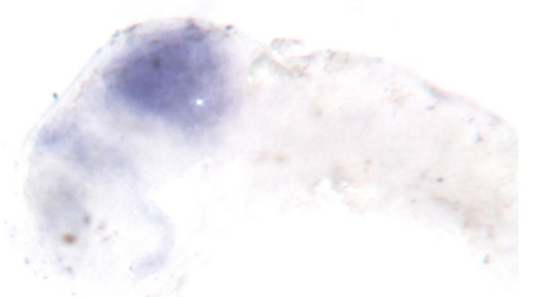

C'

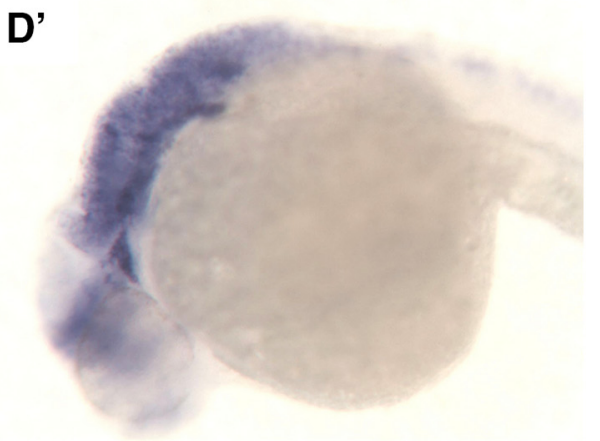

Figure 6. Early development of CNS is normal in homozygous nox2/cybb mutants. In situ hybridization with probes for marker genes of CNS patterning in $36 \mathrm{hpf} \mathrm{WT} \mathrm{and} \mathrm{nox2/cybb}{ }^{-1-}$ mutant embryos. $\boldsymbol{A}-\boldsymbol{A}^{\prime}$, pax6a expression was found in the telencephalon (TC), lens, retina, and hindbrain (HB) in both WT and nox2/cybb-1- embryos. otx2 expression was detected in midbrain (MB; $\left.\boldsymbol{B}-\boldsymbol{B}^{\prime}\right)$ and fgf8 expression at the midbrain-hindbrain boundary $\left(\boldsymbol{C}-\boldsymbol{C}^{\prime}\right)$ in both WT and mutant embryos. Eyes and yolk were removed for better visualization of otx2 expression in $\boldsymbol{B}-\boldsymbol{B}^{\prime}$. The development of major brain regions was not affected in nox $2 / c y b b^{-1-}$ embryos. $\boldsymbol{D}-\boldsymbol{D}^{\prime}$, Expression pattern of nestin, a marker for neural progenitor cells, was similar in WT and mutant embryos and nestin expression was found in all major brain regions. Scale bar, $200 \mu \mathrm{m}$.

Fig. $8 D, E)$. Growth cone $\mathrm{H}_{2} \mathrm{O}_{2}$ levels were not significantly different in RGC neurons derived from nox2/cybb ${ }^{-1-}$ mutants compared with WT neurons ( $p=0.46$; Mann-Whitney test; Fig. $8 F$ ). The fact that the $\mathrm{H}_{2} \mathrm{O}_{2}$ levels were not significantly different between WT and mutant embryos and cultured RGC neurons could be explained by compensatory mechanisms and does not eliminate the possibility that the nox $2 / c y b b^{-1-}$ mutants exhibit different levels in earlier stages of development. 

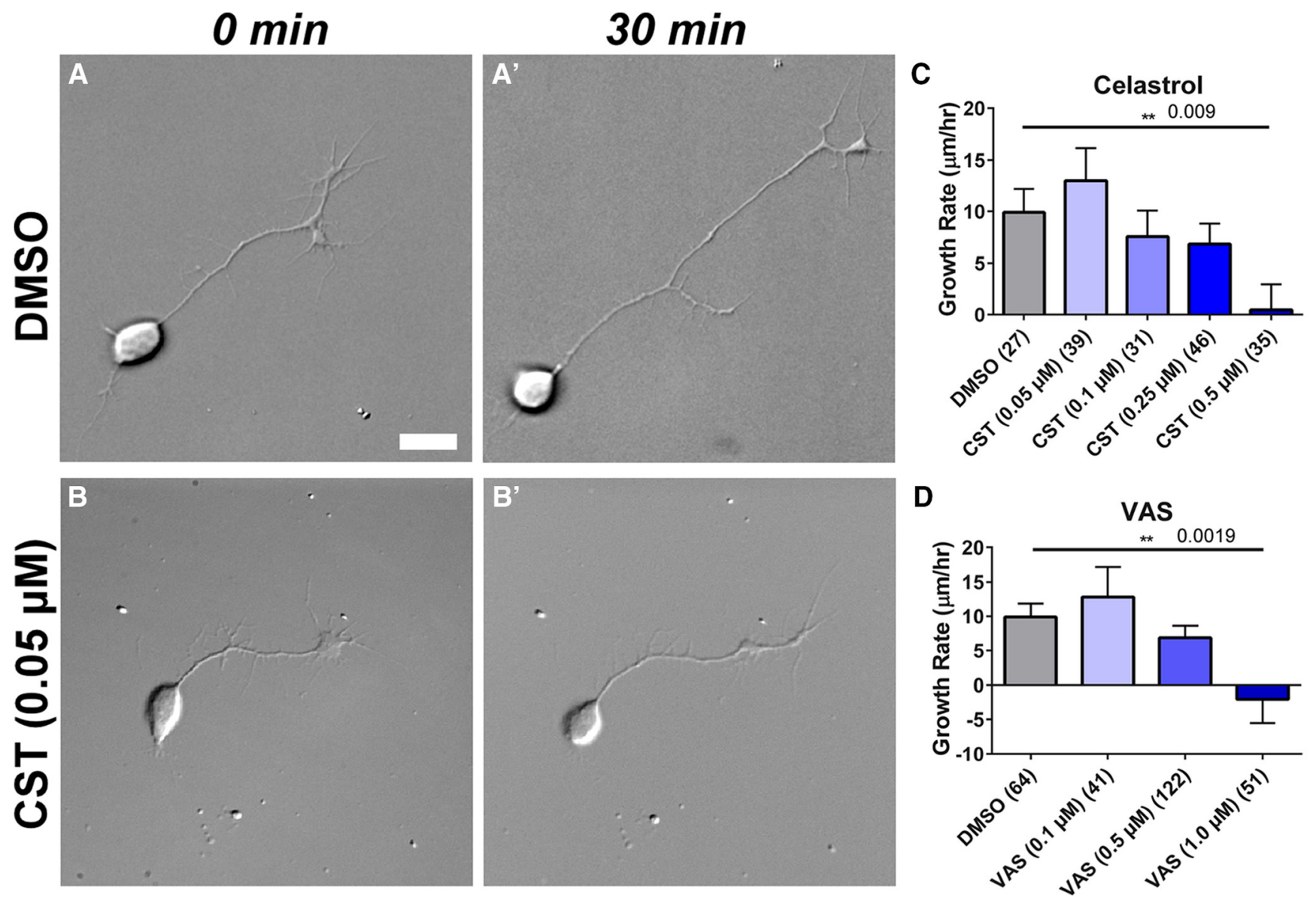

Figure 7. Nox inhibitors decrease neurite outgrowth in cultured zebrafish RGCS. $A$, Cultured zebrafish RGC neuron from a $36 \mathrm{hpf} T g($ ath5:GFP) embryos shown in differential interference contrast at $0 \mathrm{~min}$ and $30 \mathrm{~min}$ later $\left(\boldsymbol{A}^{\prime}\right)$. Scale bar, $10 \mu \mathrm{m}$. $\boldsymbol{B}$, Different neuron in the presence of $0.05 \mu \mathrm{m}$ celastrol and $30 \mathrm{~min}$ later $\left(\boldsymbol{B}^{\prime}\right)$. Axon continued to grow, although not as fast as in control condition. C, Graph of neurite outgrowth in response to $0.1 \%$ DMSO (control) or increasing concentrations of celastrol. Numbers in parentheses indicate the number of cells analyzed. Graphs show mean \pm SEM. Only $0.5 \mu$ m celastrol reduced neurite outgrowth rate significantly compared with control ( $p=0.009$; unpaired Student's $t$ test). $D$, Graph of neurite outgrowth in response to $0.1 \%$ DMSO (control) or different concentrations of VAS2870. Only $1 \mu \mathrm{M}$ VAS2870 reduced neurite outgrowth rate significantly compared with control condition ( $p=0.0019$; unpaired Student's $t$ test).

\section{Discussion}

There is emerging evidence that NADPH oxidases play a role during nervous system development (Wilson et al., 2017); however, the detailed functions of individual Nox isoforms in neuronal development are not well understood, especially in vivo. We have shown previously in cultured A. californica neurons that cell-autonomous Nox activity is critical for proper actin organization, actin retrograde flow, and neurite outgrowth (Munnamalai and Suter, 2009; Munnamalai et al., 2014). Here, we investigated the role of Nox in zebrafish retinotectal development. We found that Nox inhibitors caused an expansion of the GCL, thinning of the ON, decreased OT innervation, and mistargeting of axons within the OT (Fig. 1). Decreased OT innervation due to pharmacological Nox inhibition in zebrafish embryos is in agreement with a recent report by another group (Gauron et al., 2016). To investigate the role of specific Nox isoforms in this process, we used CRISPR/Cas9 mutagenesis to create chimeric mutants targeted to each of four zebrafish nox isoforms. Among all four nox isoform mutants, we observed that nox $2 / c y b b$ and nox 5 chimeric mutant fish displayed a phenotype that was most similar to the one caused by pharmacological Nox inhibition (Fig. 2). However, nox5 mutants frequently display early developmental problems, so we focused our study on Nox2. nox $2 / c y b b$ chimeric mutants exhibited ON thinning and decreased tectal innervation, which could be rescued by treatment with $\mathrm{H}_{2} \mathrm{O}_{2}$.
Whereas the thinning of the $\mathrm{ON}$ in the nox2/cybb chimeric mutants recovered by $72 \mathrm{hpf}$, the area of tectal innervation remained reduced by 120 hpf, suggesting a key role of Nox 2 signaling in tectal innervation (Fig. 3).

To better characterize the impact of nox2/cybb mutation, we then bred chimeric mutants into stable lines carrying a truncated version of Nox2/Cybb for each of the two nox $2 / c y b b$-specific gRNAs $\left(\right.$ nox $\left.2 / c y b b^{-1-}\right)$. Both stable homozygous nox $2 / c y b b^{-1-}$ mutant fish lines derived from either gRNA I or gRNA II showed incomplete penetrance of the phenotype. For both lines, we found that $25 \%$ of the progeny exhibited the mutant phenotype $(p<0.0001$, paired $t$ test), which is consistent across generations. The remaining $75 \%$ of the embryos were not significantly different from WT littermates with respect to development, GCL, and OT phenotype. Sequencing confirmed the genotype of homozygous nox2/cybb $b^{-1-}$ embryos and off-target genes were not affected. The incomplete phenotypic penetrance could be due to compensation by other Nox isoforms. Homozygous nox 2/ $c y b b^{-1-}$ mutants displayed GCL expansion, alterations in retinal morphology, and mistargeting of axons in the OT, forebrain, and spinal cord (Figs. 4, 5). None of these phenotypes improve at later stages, suggesting that they are not simply the result of developmental delays. The early brain development of these mutants is normal, suggesting that the axonal phenotypes are specific to loss of Nox2 and not due to secondary effects because of defects in 


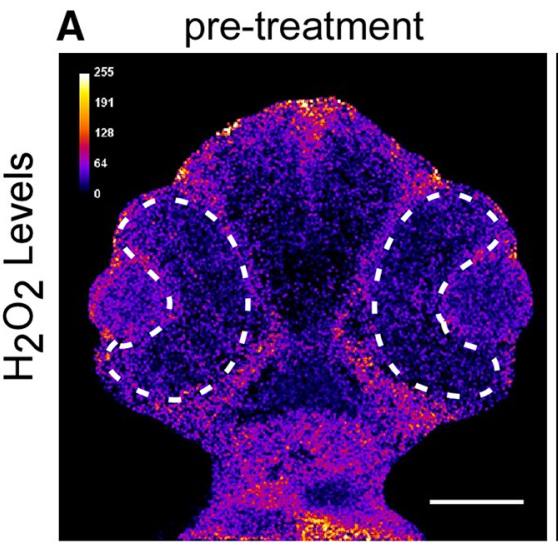

B

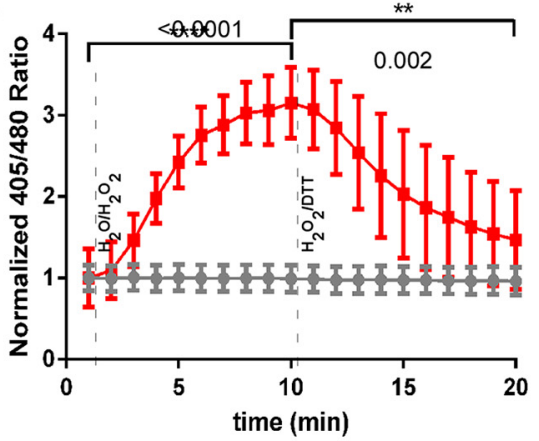

$+20 \mathrm{mM} \mathrm{H}_{2} \mathrm{O}_{2}$

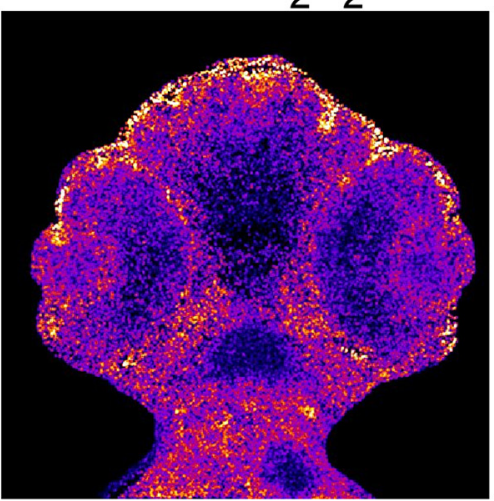

$\leadsto \mathrm{H}_{2} \mathrm{O}(\mathrm{n}=7)$

$\rightarrow-\mathrm{H}_{2} \mathrm{O}_{2} / \mathrm{DTT}(\mathrm{n}=5)$
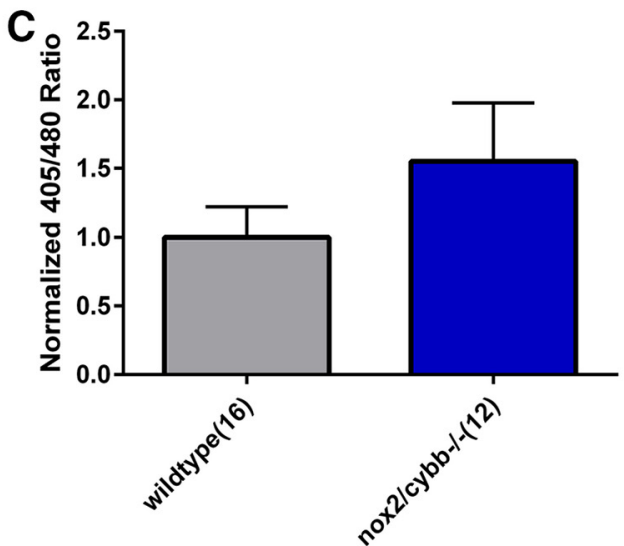

nox $2 /$ cybb-/-

$+100 \mu \mathrm{M} \mathrm{H}_{2} \mathrm{O}_{2}$

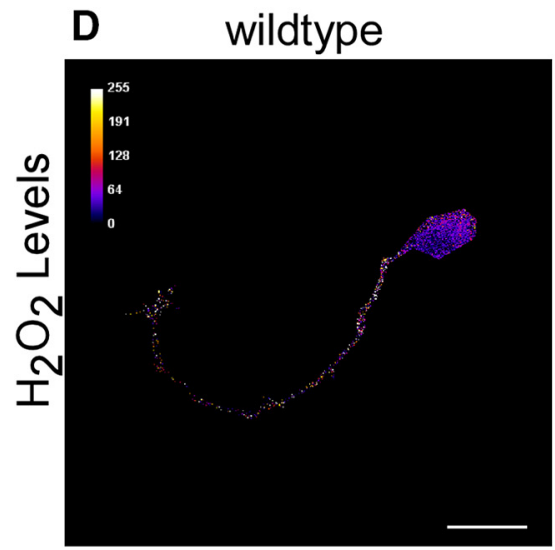

E

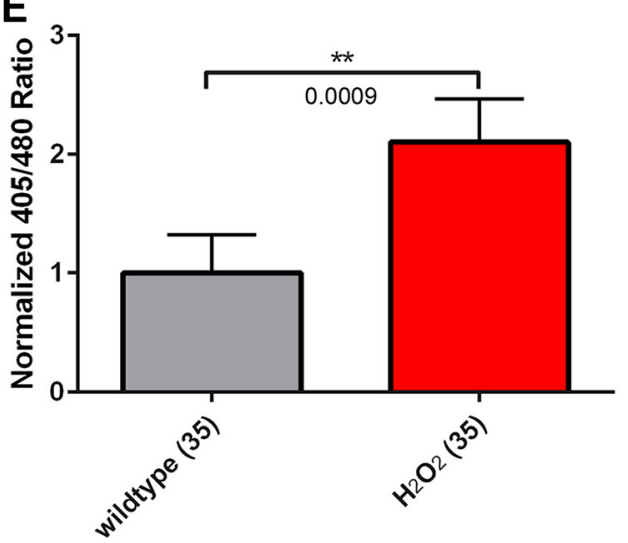

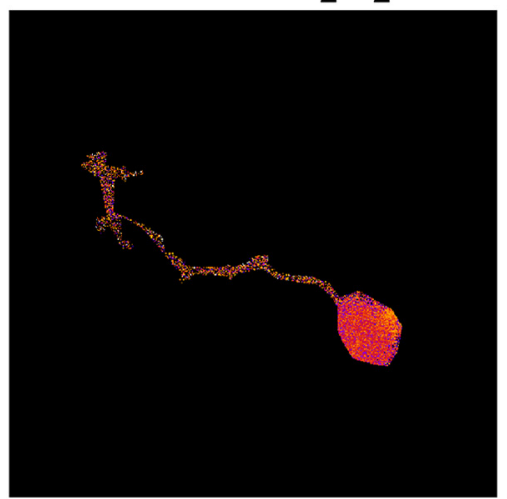

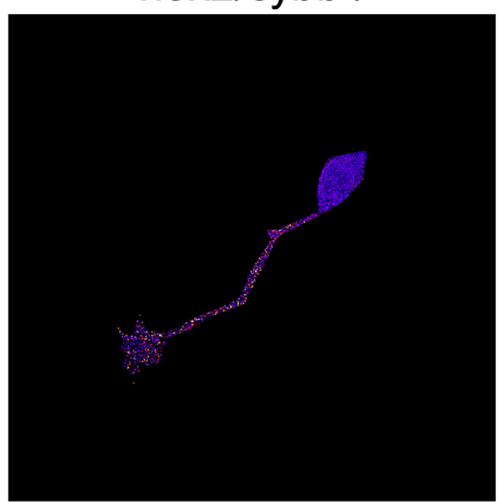

F

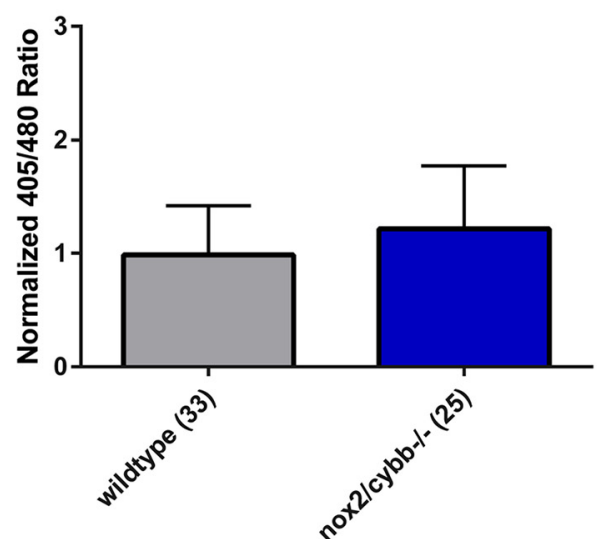

Figure 8. $\mathrm{H}_{2} \mathrm{O}_{2}$ levels in retinas of homozygous nox $2 / \mathrm{cybb}^{-1-}$ mutants. $A$, Ratiometric images depicting $\mathrm{H}_{2} \mathrm{O}_{2}$ levels in the head of a $48 \mathrm{hpfWT}$ zebrafish embryo expressing roGFP2-orp1, before (left), 10 min after $20 \mathrm{~mm} \mathrm{H}_{2} \mathrm{O}_{2}$ (middle), and 10 min after $2.5 \mathrm{~mm}$ DTT (right) addition. Dashed white line demarks retina, in which $\mathrm{H}_{2} \mathrm{O}_{2}$ levels were quantified in $\boldsymbol{B}$ and $\boldsymbol{C}$. Scale bar: $100 \mu \mathrm{m}$. $\boldsymbol{B}, \mathrm{Graph}$ showing the normalized 405/480 ratios of WT embryos at 1 min intervals in response to either $\mathrm{H}_{2} \mathrm{O}_{2} / \mathrm{DTT}$ (red line; $n=5$ embryos) or $\mathrm{H}_{2} \mathrm{O}$ (gray line; $n=7$ embryos) addition. Data are presented as mean \pm SEM. $p$-values are indicated for paired $t$ tests between $\mathrm{H}_{2} \mathrm{O}$ and $\mathrm{H}_{2} \mathrm{O}_{2}(p<0.0001)$ and $\mathrm{H}_{2} \mathrm{O}_{2}$ and DTT $(p=0.002)$. C, Bar graph showing the basal $\mathrm{H}_{2} \mathrm{O}_{2}$ (Figure legend continues.) 
early nervous system development (Fig. 6). Because Nox2 has been implicated in neural stem cell/progenitor cell maintenance in mice (Dickinson et al., 2011; Le Belle et al., 2011; Nayernia et al., 2017), we investigated whether nox $2 / c y b b^{-1-}$ mutants embryos exhibit a difference in expression of nestin, a marker of neural stem cell/progenitor cell in the brain. We did not find any obvious difference in nestin expression, suggesting that neurogenesis is not generally affected in nox $2 / c y b b^{-1-}$ mutants (Fig. 6D).

We can divide the phenotypes observed in this study into two broad classes. The first class includes $\mathrm{ON}$ thinning and a decrease in the area of OT innervation. Because both of these phenotypes respond to $\mathrm{H}_{2} \mathrm{O}_{2}$, they may be generally sensitive to ROS and not absolutely dependent on Nox2/Cybb. Another potential explanation of this phenotype could be an incomplete reduction of Nox $2 / C y b b$ activity in the chimeric mutant, which may not trigger a compensation reaction by other Nox isoforms. Phenotypes of the second class did not respond to the addition of $\mathrm{H}_{2} \mathrm{O}_{2}$ and may be highly specific to Nox2 function. These include GCL expansion and the mistargeting of axons within the OT. The widening of the GCL could be due to the fact that RGCs exit the cell cycle and differentiate earlier compared with WT embryos. In the OT, axons reach the midbrain without delay, but extend outside of the presumptive OT (Figs. 4, 5). The absence of $\mathrm{H}_{2} \mathrm{O}_{2}$ mediated rescue for this second class of phenotypes suggests that they specifically require Nox2/Cybb and alternative sources of ROS will not suffice. HyPer imaging in developing zebrafish revealed that $\mathrm{H}_{2} \mathrm{O}_{2}$ levels are very high during the first $48 \mathrm{hpf}$ in the CNS, especially in the OT (Gauron et al., 2016), further supporting an important role of $\mathrm{H}_{2} \mathrm{O}_{2}$ in early nervous system development. Therefore, we speculate that the homozygous mutants exhibiting the phenotype cannot compensate the lack of $\mathrm{H}_{2} \mathrm{O}_{2}$ in the earlier stages of development. At $48 \mathrm{hpf}$, we found no significant difference in $\mathrm{H}_{2} \mathrm{O}_{2}$ levels in the retinas of mutant embryos compared with WT embryos (Fig. 8). Therefore, $\mathrm{H}_{2} \mathrm{O}_{2}$ levels are very critical during the first $2 \mathrm{~d}$ of development and embryonic development worsens progressively if reduced $\mathrm{H}_{2} \mathrm{O}_{2}$ levels cannot be compensated by other ROS sources during the first $48 \mathrm{hpf}$.

Embryos injected with CRISPR/Cas9 components display chimerism due to the time required for CRISPR/Cas 9 complex formation after injection. During this time, cells of the embryo continue to divide, leading to the generation of unique mutations in each cell. This results in chimeric animals carrying multiple mutations along with WT alleles. Because the type of mutation generated at the target site is variable, it is impossible to determine the net result on protein function. This means that chimeras typically have less severe phenotypes because of the presence of an unknown percentage of functional enzyme. nox $2 / c y b b$ chimeric mutants express both functional and nonfunctional Nox2/ Cybb, leading to a global decrease in Nox2/Cybb activity. This means that Nox2/Cybb-dependent events such as GCL develop-

\footnotetext{
(Figure legend continued.) levels in retinas of WT $(n=16)$ and nox2/cybb ${ }^{-1-}$ mutant $(n=$ 12) embryos. nox $2 / \mathrm{Cybb}^{-1-}$ mutant embryos do not have significantly different $\mathrm{H}_{2} \mathrm{O}_{2}$ levels compared with WT embryos ( $p=0.2675$, unpaired $t$ test with Welch's correction). $\boldsymbol{D}$, Representative ratiometric images of cultured WT RGC neurons treated with serum-free control medium (left), treated for 30 min with $100 \mu \mathrm{M} \mathrm{H}_{2} \mathrm{O}_{2}$ (middle), and cultured nox2/cybb mutant RGC neuron (right). Scale bar, $10 \mu \mathrm{m}$. $\boldsymbol{E}$, Quantification of normalized $405 / 480$ ratio in growth cones of WT RGCs before and 30 min after treatment with $100 \mu \mathrm{M} \mathrm{H}_{2} \mathrm{O}_{2}$, which caused significant increase in growth cone $\mathrm{H}_{2} \mathrm{O}_{2}$ levels. Data are presented as mean \pm SEM; $n=35$ indicates number of growth cones; $p=0.0009$; Wilcoxon matched pairs test. $F$, Growth cone $\mathrm{H}_{2} \mathrm{O}_{2}$ levels are not significantly different between WT $(n=33)$ and nox2/cybb ${ }^{-1-}(n=25)$ $\operatorname{RGC}$ neurons ( $p=0.46$; Mann-Whitney test).
}

ment and targeting of axons in the OT still occur, but may happen more slowly. This could explain why $\mathrm{ON}$ thinning and decreased OT innervation partially recovered at later time points in chimeric mutants.

Conversely, nox $2 / c y b b^{-1-}$ embryos are not expected to express any functional protein and thus experience a strong, persistent loss of Nox2/Cybb activity. Given that Nox isoforms are known to compensate for one another, it is reasonable to speculate that $n o x 2 / c y b b^{-1-}$ mutants might activate compensatory mechanisms to survive. These mechanisms may include increased expression of other Nox isoforms, a decrease in cellular antioxidants, or activated regulatory subunits binding to a different Nox isoform (Yang et al., 2001; Bánfi et al., 2003; Frantz et al., 2006; Pendyala and Natarajan, 2010). Compensation by other Nox isoforms in the nox $2 / c y b b^{-1-}$ embryos could explain the fact that $\mathrm{H}_{2} \mathrm{O}_{2}$ levels were not reduced at the time points measured and the incomplete penetrance of the phenotype. Any of these alternative sources of ROS would be expected to mask phenotypes in much the same way that $\mathrm{H}_{2} \mathrm{O}_{2}$ can rescue these phenotypes in chimeric mutants. This fits our observations because nox $2 / c y b b^{-1-}$ mutants do not exhibit $\mathrm{ON}$ thinning or a decrease in OT innervation. Compensatory sources of ROS, for example, Nox1, could explain the increased $\mathrm{H}_{2} \mathrm{O}_{2}$ levels in nox $2 / c y b b^{-1-}$ mutants, which might cause the mistargeting of axons in the OT, as opposed to the reduced tectal innervation found in $n o x 2 / c y b b$ chimeric mutants. Compensation to mask developmental delays is not expected in chimeras because functional Nox2/Cybb is still present in these embryos.

Differences in the timing and extent of interfering with Nox activity in the approaches used in our study might also explain the observed response to pharmacological Nox inhibition. Celastrol treatment is expected to inhibit completely all Nox isoforms temporally restricted to a few hours during ON development. These embryos display phenotypes of both classes such as GCL expansion, $\mathrm{ON}$ thinning, and a decrease in the area of OT innervation likely stemming from the temporary but complete loss of Nox activity. Whereas our in vivo results clearly suggest a function for Nox2/Cybb in retinal development, we cannot infer a purely cellautonomous role for Nox2 signaling in RGCs for all observed phenotypes using our current approaches in whole embryos. However, neurite outgrowth in dissociated RGC cultures was decreased by celastrol and VAS2870 in a dose-dependent manner, suggesting that RGCs require cell-autonomous Nox activity for proper outgrowth (Fig. 7).

Our current approach of CRISPR/Cas9 mutagenesis does not allow for spatial or temporal control. However, newly developed techniques allow for restricted expression of Cas9 using cell-typespecific (Ablain et al., 2015) or heat-shock promoters (Yin et al., 2015). Cell-type-specific mutagenesis will provide insight into cell-autonomous functions of Nox, whereas temporally controlled mutagenesis will reveal the role of Nox at specific developmental stages. In addition, these new technologies will allow for the dissection of molecular signaling pathways in specific cell types that underlie the phenotypes that we report here. In conclusion, we report that Nox2/Cybb is critically involved in the development of RGCs in the zebrafish retina and the development of retinotectal connections.

\section{References}

Ablain J, Durand EM, Yang S, Zhou Y, Zon LI (2015) A CRISPR/Cas9 vector system for tissue-specific gene disruption in zebrafish. Dev Cell 32:756764. CrossRef Medline

Avanesov A, Malicki J (2010) Analysis of the retina in the zebrafish model. Methods Cell Biol 100:153-204. CrossRef Medline 
Bánfi B, Clark RA, Steger K, Krause KH (2003) Two novel proteins activate superoxide generation by the NADPH oxidase NOX1. J Biol Chem 278: 3510-3513. CrossRef Medline

Bishop B, Ho KK, Tyler K, Smith A, Bonilla S, Leung YF, Ogas J (2015) The chromatin remodeler chd5 is necessary for proper head development during embryogenesis of danio rerio. Biochim Biophys Acta 1849:10401050. CrossRef Medline

Brandes RP, Weissmann N, Schröder K (2014) Nox family NADPH oxidases: molecular mechanisms of activation. Free Rad Biol Med 76:208226. CrossRef Medline

Brown DI, Griendling KK (2009) Nox proteins in signal transduction. Free Rad Biol Med 47:1239-1253. CrossRef Medline

Burrill JD, Easter SS Jr (1995) The first retinal axons and their microenvironment in zebrafish: cryptic pioneers and the pretract. J Neurosci 15: 2935-2947. CrossRef Medline

Chen Z, Lee H, Henle SJ, Cheever TR, Ekker SC, Henley JR (2013) Primary neuron culture for nerve growth and axon guidance studies in zebrafish (Danio rerio). PLoS One 8:e57539. CrossRef Medline

Choi DH, Cristóvão AC, Guhathakurta S, Lee J, Joh TH, Beal MF, Kim YS (2012) NADPH oxidase 1-mediated oxidative stress leads to dopamine neuron death in Parkinson's disease. Antioxid Redox Signal 16:10331045. CrossRef Medline

Coyoy A, Olguín-Albuerne M, Martínez-Briseño P, Moran J (2013) Role of reactive oxygen species and $\mathrm{NADPH}$-oxidase in the development of rat cerebellum. Neurochem Int 62:998-1011. CrossRef Medline

De Pasquale R, Beckhauser TF, Hernandes MS, Giorgetti Britto LR (2014) LTP and LTD in the visual cortex require the activation of NOX2. J Neurosci 34:12778-12787. CrossRef Medline

Dickinson BC, Peltier J, Stone D, Schaffer DV, Chang CJ (2011) Nox2 redox signaling maintains essential cell populations in the brain. Nat Chem Biol 7:106-112. CrossRef Medline

Dvoriantchikova G, Grant J, Santos AR, Hernandez E, Ivanov D (2012) Neuronal NAD $(\mathrm{P}) \mathrm{H}$ oxidases contribute to ROS production and mediate RGC death after ischemia. Invest Ophthalmol Vis Sci 53:2823-2830. CrossRef Medline

Frantz S, Brandes RP, Hu K, Rammelt K, Wolf J, Scheuermann H, Ertl G, Bauersachs J (2006) Left ventricular remodeling after myocardial infarction in mice with targeted deletion of the NADPH oxidase subunit gp91PHOX. Basic Res Cardiol 101:127-132. CrossRef Medline

Gauron C, Meda F, Dupont E, Albadri S, Quenech'Du N, Ipendey E, Volovitch M, Del Bene F, Joliot A, Rampon C, Vriz S (2016) Hydrogen peroxide (H2O2) controls axon pathfinding during zebrafish development. Dev Biol 414:133-141. CrossRef Medline

Gutscher M, Sobotta MC, Wabnitz GH, Ballikaya S, Meyer AJ, Samstag Y, Dick TP (2009) Proximity-based protein thiol oxidation by $\mathrm{H} 2 \mathrm{O} 2$ scavenging peroxidases. J Biol Chem 284:31532-31540. CrossRef Medline

Hensley MR, Leung YF (2010) A convenient dry feed for raising zebrafish larvae. Zebrafish 7:219-231. CrossRef Medline

Heumüller S, Wind S, Barbosa-Sicard E, Schmidt HH, Busse R, Schröder K, Brandes RP (2008) Apocynin is not an inhibitor of vascular NADPH oxidases but an antioxidant. Hypertension 51:211-217. CrossRef Medline

Hu M, Easter SS (1999) Retinal neurogenesis: the formation of the initial central patch of postmitotic cells. Dev Biol 207:309-321. CrossRef Medline

Jao LE, Wente SR, Chen W (2013) Efficient multiplex biallelic zebrafish genome editing using a CRISPR nuclease system. Proc Natl Acad Sci U S A 110:13904-13909. CrossRef Medline

Jaquet V, Marcoux J, Forest E, Leidal KG, McCormick S, Westermaier Y, Perozzo R, Plastre O, Fioraso-Cartier L, Diebold B, Scapozza L, Nauseef WM, Fieschi F, Krause KH, Bedard K (2011) NADPH oxidase (NOX) isoforms are inhibited by celastrol with a dual mode of action. Br J Pharmacol 164:507-520. CrossRef Medline

Kajla S, Mondol AS, Nagasawa A, Zhang Y, Kato M, Matsuno K, YabeNishimura C, Kamata T (2012) A crucial role for nox 1 in redoxdependent regulation of wnt-beta-catenin signaling. FASEB J 26: 2049-2059. CrossRef Medline

Kawahara T, Lambeth JD (2007) Molecular evolution of phox-related regulatory subunits for NADPH oxidase enzymes. BMC Evol Biol 7:178. CrossRef Medline

Kawahara T, Quinn MT, Lambeth JD (2007) Molecular evolution of the reactive oxygen-generating NADPH oxidase (Nox/Duox) family of enzymes. BMC Evol Biol 7:109. CrossRef Medline
Kimmel CB, Ballard WW, Kimmel SR, Ullmann B, Schilling TF (1995) Stages of embryonic development of the zebrafish. Dev Dyn 203:253-310. CrossRef Medline

Kishida KT, Hoeffer CA, Hu D, Pao M, Holland SM, Klann E (2006) Synaptic plasticity deficits and mild memory impairments in mouse models of chronic granulomatous disease. Mol Cell Biol 26:5908-5920. CrossRef Medline

Kita EM, Scott EK, Goodhill GJ (2015a) Topographic wiring of the retinotectal connection in zebrafish. Dev Neurobiol 75:542-556. CrossRef Medline

Kita EM, Scott EK, Goodhill GJ (2015b) The influence of activity on axon pathfinding in the optic tectum. Dev Neurobiol 75:608-620. CrossRef Medline

Le Belle JE, Orozco NM, Paucar AA, Saxe JP, Mottahedeh J, Pyle AD, Wu H, Kornblum HI (2011) Proliferative neural stem cells have high endogenous ROS levels that regulate self-renewal and neurogenesis in a PI3K/ Akt-dependent manner. Cell Stem Cell 8:59-71. CrossRef Medline

Li Z, Ptak D, Zhang L, Walls EK, Zhong W, Leung YF (2012) Phenylthiourea specifically reduces zebrafish eye size. PLoS One 7:e40132. CrossRef Medline

Mahler J, Driever W (2007) Expression of the zebrafish intermediate neurofilament nestin in the developing nervous system and in neural proliferation zones at postembryonic stages. BMC Dev Biol 7:89. CrossRef Medline

Meeker ND, Hutchinson SA, Ho L, Trede NS (2007) Method for isolation of PCR-ready genomic DNA from zebrafish tissues. Biotechniques 43:610, 612, 614. CrossRef Medline

Munnamalai V, Suter DM (2009) Reactive oxygen species regulate F-actin dynamics in neuronal growth cones and neurite outgrowth. J Neurochem 108:644-661. CrossRef Medline

Munnamalai V, Weaver CJ, Weisheit CE, Venkatraman P, Agim ZS, Quinn MT, Suter DM (2014) Bidirectional interactions between NOX2-type NADPH oxidase and the F-actin cytoskeleton in neuronal growth cones. J Neurochem 130:526-540. CrossRef Medline

Nayernia Z, Jaquet V, Krause KH (2014) New insights on NOX enzymes in the central nervous system. Antioxid Redox Signal 20:2815-2837. CrossRef Medline

Nayernia Z, Colaianna M, Robledinos-Antón N, Gutzwiller E, Sloan-Béna F, Stathaki E, Hibaoui Y, Cuadrado A, Hescheler J, Stasia MJ, Saric T, Jaquet V, Krause KH (2017) Decreased neural precursor cell pool in NADPH oxidase 2 deficiency: from mouse brain to neural differentiation of patient derived iPSC. Redox Biol 13:82-93. CrossRef Medline

Olguin-Albuerne M, Moran J (2015) ROS produced by NOX2 control in vitro development of cerebellar granule neurons development. ASN Neuro 7: pii: 1759091415578712. CrossRef Medline

Pendyala S, Natarajan V (2010) Redox regulation of nox proteins. Respir Physiol Neurobiol 174:265-271. CrossRef Medline

Poggi L, Vitorino M, Masai I, Harris WA (2005) Influences on neural lineage and mode of division in the zebrafish retina in vivo. J Cell Biol 171:991-999. CrossRef Medline

Ren F, Wang K, Zhang T, Jiang J, Nice EC, Huang C (2015) New insights into redox regulation of stem cell self-renewal and differentiation. Biochim Biophys Acta 1850:1518-1526. CrossRef Medline

Rieger S, Sagasti A (2011) Hydrogen peroxide promotes injury-induced peripheral sensory axon regeneration in the zebrafish skin. PLoS Biol 9:e1000621. CrossRef Medline

Rosowski EE, Deng Q, Keller NP, Huttenlocher A (2016) Rac2 functions in both neutrophils and macrophages to mediate motility and host defense in larval zebrafish. J Immunol 197:4780-4790. CrossRef Medline

Schneider CA, Rasband WS, Eliceiri KW (2012) NIH image to ImageJ: 25 years of image analysis. Nat Methods 9:671-675. CrossRef Medline

Spencer NY, Zhou W, Li Q, Zhang Y, Luo M, Yan Z, Lynch TJ, Abbott D, Bánfi B, Engelhardt JF (2013) Hepatocytes produce TNF-alpha following hypoxia-reoxygenation and liver ischemia-reperfusion in a NADPH oxidase- and c-src-dependent manner. Am J Physiol Gastrointest Liver Physiol 305:G84-G94. CrossRef Medline

Stenkamp DL (2015) Development of the vertebrate eye and retina. Prog Mol Biol Transl Sci 134:397-414. CrossRef Medline

Suter DM (2011) Live cell imaging of neuronal growth cone motility and guidance in vitro. Methods Mol Biol 769:65-86. CrossRef Medline

Tauzin S, Starnes TW, Becker FB, Lam PY, Huttenlocher A (2014) Redox 
and src family kinase signaling control leukocyte wound attraction and neutrophil reverse migration. J Cell Biol 207:589-598. CrossRef Medline

Tejada-Simon MV, Serrano F, Villasana LE, Kanterewicz BI, Wu GY, Quinn MT, Klann E (2005) Synaptic localization of a functional NADPH oxidase in the mouse hippocampus. Mol Cell Neurosci 29:97-106. CrossRef Medline

Weaver CJ, Leung YF, Suter DM (2016) Expression dynamics of NADPH oxidases during early zebrafish development. J Comp Neurol 524:2130 2141. CrossRef Medline

Weaver JR, Taylor-Fishwick DA (2013) Regulation of NOX-1 expression in beta cells: a positive feedback loop involving the src-kinase signaling pathway. Mol Cell Endocrinol 369:35-41. CrossRef Medline

Westerfield M (2000) The zebrafish book: a guide for the laboratory use of zebrafish (Danio rerio), Ed 4. Eugene, OR: University of Oregon.

Wilkinson RN, Elworthy S, Ingham PW, van Eeden FJ (2013) A method for high-throughput PCR-based genotyping of larval zebrafish tail biopsies. Biotechniques 55:314-316. CrossRef Medline

Wilson C, Núñez MT, González-Billault C (2015) Contribution of NADPH oxidase to the establishment of hippocampal neuronal polarity in culture. J Cell Sci 128:2989-2995. CrossRef Medline

Wilson C, Muñoz-Palma E, Henríquez DR, Palmisano I, Núñez MT, Di Giovanni S, González-Billault C (2016) A feed-forward mechanism involv- ing the NOX complex and RyR-mediated Ca2 + release during axonal specification. J Neurosci 36:11107-11119. CrossRef Medline

Wilson C, Munoz-Palma E, Gonzalez-Billault C (2017) From birth to death: A role for reactive oxygen species in neuronal development. Semin Cell Dev Biol pii: S1084-9521(17)30334-8. CrossRef Medline

Yang S, Madyastha P, Bingel S, Ries W, Key L (2001) A new superoxidegenerating oxidase in murine osteoclasts. J Biol Chem 276:5452-5458. CrossRef Medline

Yin L, Maddison LA, Li M, Kara N, LaFave MC, Varshney GK, Burgess SM, Patton JG, Chen W (2015) Multiplex conditional mutagenesis using transgenic expression of Cas9 and sgRNAs. Genetics 200:431-441. CrossRef Medline

Yoo SK, Starnes TW, Deng Q, Huttenlocher A (2011) Lyn is a redox sensor that mediates leukocyte wound attraction in vivo. Nature 480:109-112. CrossRef Medline

Yoo SK, Freisinger CM, LeBert DC, Huttenlocher A (2012) Early redox, Src family kinase, and calcium signaling integrate wound responses and tissue regeneration in zebrafish. J Cell Biol 199:225-234. CrossRef Medline

Zhu X, Xu Y, Yu S, Lu L, Ding M, Cheng J, Song G, Gao X, Yao L, Fan D, Meng S, Zhang X, Hu S, Tian Y (2014) An efficient genotyping method for genome-modified animals and human cells generated with CRISPR/Cas9 system. Sci Rep 4:6420. CrossRef Medline 